\title{
Grey Wolf based Wang's Demons for Retinal Image Registration
}

\author{
Sayan Chakraborty ${ }^{1}$, Ratika Pradhan², Amira S. Ashour 3 , Luminita Moraru', Nilanjan Dey ${ }^{5}$ \\ 1 Dept. of CA, Sikkim Manipal Institute of Technology, sayan.cb@gmail.com \\ 2 Dept. of CA, Sikkim Manipal Institute of Technology, ratika.p@smit.smu.edu.in \\ 3 Department of Electronics and Electrical Communications Engineering, Faculty of Engineering, Tanta Univ., \\ Egypt, amirasashour@yahoo.com \\ 4 Department of Chemistry, Physics \& Environment, Faculty of Sciences and Environment, Dunarea de Jos \\ University of Galati, 47 Domneasca Str., 800008 Galati, Romania; Luminita.Moraru@ugal.ro \\ 5 Department of Information Technology, Techno India College of Technology, West Bengal, 740000, India, \\ neelanjan.dey@gmail.com \\ * Preprint version
}

\begin{abstract}
Image registration has an imperative role in medical imaging. In this work, a grey-wolf optimizer (GWO) based non-rigid demons registration is proposed to support the retinal image registration process. A comparative study of the proposed GWO-based demons registration framework with cuckoo search, firefly algorithm, and particle swarm optimization- based demons registration is conducted. In addition, a comparative analysis of different demons registration methods, such as Wang's demons, Tang's demons, and Thirion's demons which are optimized using the proposed GWO is carried out. The results established the superiority of the GWO-based framework which achieved 0.9977 correlation, and fast processing compared to the use of the other optimization algorithms. Moreover, GWO-based Wang's demons performed better accuracy compared to the Tang's demons and Thirion's demons framework. It also achieved the best less registration error of $8.36 \times 10^{-5}$.
\end{abstract}

Keywords: Demons registration, firefly algorithm, cuckoo search, grey-wolf optimization, correlation, image registration.

\section{Introduction}

Retinal image registration [1,2] has an essential role to assist the ophthalmologist in the diagnosis of eye diseases, evaluate the disease and their growth rate, screen the patient's screening, and to assist laser surgical procedures. The blood vessels in the retina [3] can be directly imaged noninvasively, such as the fundus images and fluorescein angiogram (FA) images [4,5]. The most common image registration methods include area-based, feature-based, and hybrid registration methods. Area-based registration is dependent mainly on the intensity of pixels. There are some optimized functions as well which play an integral part in this case, such as mean square error, mutual information [6], similarity measurement [7], correlation (cross and phase) [8], etc. Statically, a window of points is compared among the input and reference images [9]. Two key points affect the performance of such a system, they are large homogeneous [10] and non-uniform. In the feature-based registration methods, the features (common structures) are usually extracted and matched from reference and sensed images, where the point correspondences are available in both images. The registration process is performed by maximizing a similarity measure computed from the correspondences [10]. The performance of feature-based methods depends on the sufficiency and reliability of the correspondences. These methods consist of various steps, including feature detection, feature matching, transform model estimation, and image resampling and transformation [11]. Fovea, optic disk $[12,13]$ and retinal blood vessels [14] are some of the key features found in fundus retinal 
images [15]. There are a large number of methods using which these features can be detected. Fundus' images brightest component [16] is the optic disk (OD). Hence, it's easier to detect pixels with a higher intensity as they have higher grayscale value. On the contrary, the fovea is the least bright part in the retinal image. A priori knowledge of the optic disk position helps to understand the gap between the fovea and optic disk. The gap [13] between the center of OD and fovea is 2.5 times the diameter of the optic disk. Vessel tracking [17], matched filters [18], morphological processing [19], pixel-based classifiers such as Neural networks helps to obtain or extract the retinal blood vessels from retinal images. The hybrid image registration algorithm includes both area and feature-based registration methods [10, 20, 21].

One of the key objectives of image registration is to get rid of image registration error and make it more efficient than the existing techniques [22] with avoiding the time complexity [1-4]. Image registration refers to aligning one or more images [23, 24] with reference to other images [5]. It depends on the concept of image transformation [6], in which an image or image matrix uses various operations, such as translation, rotation, scaling, shearing, [25] and mapping. Based on such operations, image registration [26, 27, 28] can be divided into two different categories: rigid and non-rigid [29]. Rigid registration [30] consists of rotation, translation, and scaling. Unlike the rigid registration which may involve various geometric transformations [31] without changing the shape of the object, the non-rigid registration [32,33] is based on the same concept but affects the shape of the objects involved in the image registration process [1]. Non-rigid registration can be applied to mono-modal as well as multimodal images, where the transformation can include the change of the object's shapes to warp [34] the retinal images [19]. For retinal image registration, non-rigid registration [35] involves 3D eye motion, and image acquisition parameters which are imprecisely known for retinal image databases. Hence it is essential to register the retinal images to find out the correct pairs, obtain the ground-truth, and identify the difference in images by overlapping them with respect to one another.

Demons registration is an efficient non-rigid image registration technique due to its fluid registration mechanism, which allows it to have much lesser distortion of objects compared to other non-rigid registrations [7]. It changes the shape of the object [8,9]. Since the registration process includes various parameters, the optimization of image registration becomes essential in different registration processes to determine the optimal parameters for superior results [16-18]. Different optimization algorithms proved their efficiency in the different medical imaging applications, including cuckoo search (CS), firefly algorithm (FA), particle swarm optimization (PSO), and Grey wolf optimization (GWO) algorithm [10, 11, 37]. In addition, the GWO algorithm is considered one of the efficient optimization [38] methods for medical image registration and fusion. For instance, the GWO was applied to identify scale selection from brain images to improve image fusion technique [12] as well as to enhance [39] the homomorphic wavelet-based fusion [13].

In the current work, the Grey wolf optimization (GWO) algorithm [40,41] is applied to build up the optimization framework to achieve an optimal solution [42] for better accuracy and faster image non-rigid demons registration [11, 22-26]. A comparative study is conducted with the cuckoo search- [10], firefly algorithm [43] and particle swarm optimization- [9] based demons registration [44, 45, 46] framework.

The following sections of the current work include the literature survey in section 2 followed by introducing the various types of demon's algorithm-based image registration in section 3 . Section 4 discusses the optimization frameworks. The proposed method is explained in section 5 , where the results are observed and analyzed in section 6 and discussed in section 7 . The conclusion of the present work is highlighted in section 8 . 


\section{Literature survey}

Retinal image registration is an essential tool in medical imaging which attracts several researchers to develop new methods. In 2012, Gharabaghi et al. [3] proposed a retinal image registration method using geometrical features by applying for non-rigid affine registration on retinal images obtained from the DRIVE database. Ramli et al. [1] proposed a feature-based retinal image registration using D-Saddle based detector to identify the feature points in retinal images obtained from the FIRE dataset. Hernandez-Matas et al. [2] designed a retinal image registration method based on the concept of the spherical shape of the human eye, where each image was warped with respect to another image using rigid registration. Later in 2017, Zhuang et al. [47] proposed a deformable image registration algorithm based on improved active demons using the length and area of connected region as a driving force to build an active demons diffusion equation. In 2018, Li et al. [4] implemented a multimodal and multivendor retinal image registration using deformable registration based on modality independent neighborhood descriptor (MIND) framework on color fundus images. To improve the demons registration of medical images, Mishra et al. [46] used the 2D image registration method on mono-modal images. To obtain the deformation fields of medical images, the velocity field of demons was evaluated. In 2016, Lan et al. [43]proposed a comparative analysis of all demons registration, which proved that Wang's demons [43] was superior in terms of performance than Thirion's and Tang's demons.

On the other hand, due to the effectiveness of the optimization algorithms [48] in the registration [49] process, several researchers employed several optimizers to develop the registration process. Zhang and $\mathrm{Wu}$ [50] applied a firefly algorithm based rigid image registration to improve the traditional rigid registration method compared to the use of particle swarm optimization and genetic algorithm as benchmarks. The firefly algorithm was applied to spatial transformation parameters and was compared with GA, PSO, and Artificial bee Colony (ABC) algorithm. The results showed that the firefly algorithm performed better than the ABC, PSO, and GA, respectively, where the computation time of FA was only 1.0683s, slower than the ABC, but yet faster than GA and PSO. Cocianu and Stan [39] developed an image registration technique based on the firefly algorithm to realize less registration error and time complexity reduction. Ayatollahi et al. [33] proposed a PSO-based multimodal brain image registration using mutual information of the original and resultant image as the fitness function. The translation, rotation, and scaling were used as the three parameters to be optimized using hybrid particle swarm optimization. The root mean square error was measured between the original and registered images as the fitness function. The PSO based framework had less error 0.496 compared to the 0.888 error when using the GA-based framework. Zhang et al. [50] discussed the effect of the FA on rigid registration by optimizing the rotational parameter of rigid registration to achieve the lowest error. Xiaogang et al. [51] applied the FA firefly algorithm for efficient image registration using mutual information as the fitness function. Moreover, Chakraborty et al. [44] used the firefly algorithm-based image registration framework that involved the optimization of Thirion's demons. Afterward, Chakraborty et al. [21] introduced a CS algorithm-based image registration compared to the FA-based demons registration. In 2018, Yogapriya et al. [48] introduced a fuzzy-based grey wolf optimization technique for the medical image retrieval system using image texture features. This system classified the images based on the features and reduced the high dimensional texture features using fuzzy-based grey wolf optimization. Recently in 2019, Asha et al. [49] presented a multimodal medical image fusion technique combined with grey wolf optimization.

From the preceding reported studies, it is obvious that the medical image registration techniques lack a proper direction of optimization [52, 53] of the problem of registration error [54, 55] and time complexity[56]. Hence, the current work aims to address such problems during image registration. 


\section{Non-rigid image registration using Demon's algorithm}

Image registration is based on the concept of image transformation of computer graphics. The basic transformations involved in registration are translation, rotation, and scaling. In this transformation, the object's shape does not change, only their viewing perspective changes from frame to frame. Hence, these types of transformations or registrations were called rigid registrations. To make the animations more realistic, a dynamic change of the frame and objects are required. Hence, mapping, shearing, and deformation become significant in the transformation. These operations not only change the object's shape, it also deformed other parts of the frames. However, the animations looked more realistic as deformation and restoration of shapes in continuous frames made the visual livelier than rigid registration. These transformations were known as non-rigid transformations. Initially, shearing and mapping were included with the rigid transformation to introduce affine registration. Later, spline curves came into play to modify the mapping of objects from frame to frame. Nueral [58,59] or brain images [60] have been a key area to apply such non-rigid registration. Nevertheless, all these non-rigid registrations majorly lacked the concept of changing the shape of the object in such a way that it becomes imperceptible by the human eye. Hence, demons registration was originated by Thirion [30], and modified by Wang et al. [61] and Tang et al. [28]. It is known as fluid registration for its superior performance over other non-rigid registrations. For image matrix transformation, it operates through displacement deviator among the involved images. Pixel velocity and edge-based forces help to obtain the displacement deviator. These are the main three demons registration that has been widely used on medical images as follows.

\subsection{Thirion's demons}

The concept of the demons registration by Thirions is based on the optical flow, where the demons force refers to the displacement vector applied by the reference image $(\mathrm{R})$ on the deformed image. The pixels in the registered/deformed image are shifted to get aligned based on the reference image. Since the demons registration process is iterative, the deformation field is the outcome of after each iteration. The objective function should have the smoothness of the alignment field as well as similarity measurement, which is defined by [30]:

$$
E(u)=\|R-F \otimes u\|^{2}+\sigma^{2}\|u\|^{2}
$$

where the reference and query images are denoted by $R$, and $F$, respectively. Also, the deformation field $u$ is updated after each iteration, where the deformation operation is mentioned as $\otimes$. The Gaussian smoothening kernel $\sigma$ is applied in demons, which is a variable parameter. To calculate the value of $u$, the following formula is applied [30, 43]:

$$
u=(\|R-F\|)\left(\frac{\nabla R}{\|\nabla R\|^{2}+\|R-F\|^{2}}\right)
$$

where $\nabla R$ is the gradient information of the reference image, where the default values of the registration parameters as defined by Thirions are listed in Table 1.

Table 1. Default values of the registration parameters in demons registration

\begin{tabular}{ccc}
\hline & 2-D filter & Sigma \\
\hline $\begin{array}{c}\text { Velocity field smoothing } \\
\text { kernel }\end{array}$ & Gaussian low pass filter & 10 pixels \\
\hline Alpha (noise) constant & $2.5 \mathrm{~dB}$ & \\
\hline
\end{tabular}




\subsection{Wang's demons}

In Wang's demons, the registration approach was changed slightly, where Wang et al. [61] modified Thirion's demons by introducing gradient information $\nabla R$ of the reference image $R$. The problem in Thirion's demons provides the deformation field information with respect to the reference image $R$. Typically, Thirion's demons is effective for small deformed problems as it collects information from deformation field. Hence, Wang's demons [2, 19, 61] introduces the gradient information managed to eradicate these problems. The equation of Wang's demons force is given by:

$$
u=(\|R-F\|)\left(\frac{\nabla R}{\|\nabla R\|^{2}+\alpha^{2}\|R-F\|^{2}}+\frac{\nabla F}{\|\nabla F\|^{2}+\alpha^{2}\|R-F\|^{2}}\right)
$$

where $\alpha$ is the noise constant which drive the force strength to be stabilized in every iteration. The alignment field is calculated by applying the Gaussian smoothening kernel in demons is $\sigma$ [30] using the following formula:

$$
E(u)=\|R-F \otimes u\|^{2}+\sigma^{2}\|u\|^{2}
$$

In the present work, the value of $\sigma$ is optimized.

\subsection{Tang's demons}

Since the analysis of the general range of parameters was still missing in demons registration, Tang et al. [4] introduced a balance coefficient $k$ in Tang's demons to solve this problem. The adaptation of the balance coefficient is managed to adjust the demons force and its strength, where the Tang's demons force is represented by $[28,43]$ :

$$
u=(\|R-F\|)\left(\frac{\nabla R}{k^{2}\|\nabla R\|^{2}+\alpha^{2}\|R-F\|^{2}}+\frac{\nabla F}{k^{2}\|\nabla F\|^{2}+\alpha^{2}\|R-F\|^{2}}\right)
$$

where $e \nabla R$ and $e \nabla F$ are the gradient field information unit vectors of the reference image (R), and fixed image (F), respectively. The Tang's demons force is given by:

$$
u=(\|R-F\|)\left(\frac{\nabla R}{k^{2}\|\nabla R\|^{2}+\alpha^{2}\|R-F\|^{2}} e_{\nabla R}+\frac{\nabla F}{k^{2}\|\nabla F\|^{2}+\alpha^{2}\|R-F\|^{2}} e_{\nabla F}\right)
$$

Thus, despite the balance coefficient $k$, Wang's demons proved its efficiency compared to the Tang's demons [43]. The unit vectors of the gradient field are managed to simplify the Thirion's demons leading to producing worse results than Wang's demons. It has been observed that without these parameters demons registration can produce better results.

\section{Optimization framework for image registration}

A Grey wolf optimization algorithm is applied in the present work to build the framework for demons registration. The main principle of the Grey Wolf Optimizer (GWO) is originated from the behaviors and characteristics of Grey Wolf [41]. The Grey wolf optimizer is one of the most advanced meta-heuristic algorithms. It is based on the adaptation of the Grey wolf's hunting mechanism. The algorithm divides the group of grey wolves into four different subgroups: Alpha $(\alpha)$, Beta $(\beta)$, Delta $(\delta)$, and Omega $(\omega)$. The hunting area is considered as the search area of the problem statement, where alpha-, beta-, and deltawolves direct the omega wolves to the favorable zone. During the iterative process, alpha, beta, and delta change their position based on the following expression [40, 41]: 


$$
\begin{aligned}
& D=|W p(t) \cdot C-W(t)| \\
& W(t+1)=W p(t)-D \cdot A
\end{aligned}
$$

where $D$ represents the gap between the prey and predator's position, $t$ is the current iteration and $W p(t)$ is the prey's position. The Grey wolf's position is denoted by $W(t)$, which is updated in equation (8), where $A$ and $C$ are determined using the following expressions [40,41]:

$$
\begin{aligned}
& A=r_{1} a \cdot 2 a \\
& C=r_{2} \cdot 2
\end{aligned}
$$

where $r_{1}$ and $r_{2}$ are two vectors having random values between 0 and 1 , while the value of $a$ varies between 0 to 2 and is gradually decreased from 2 to 0 . Hence, the first three best solutions [40,41] values (Alpha $(\alpha)$, Beta $(\beta)$, Delta $(\delta)$ ) are initialized and updated. These values assist other search agents, including Omegas $(\omega)$ to update their positions according to the position of the best search agents. Accordingly, the following formulas help to calculate and update the position of grey wolves [40, 41]:

$$
\begin{aligned}
& D_{\alpha}=\left|C_{1} \cdot W_{\alpha}-W\right|, D_{\beta}=\left|C_{2} \cdot W_{\beta}-W\right|, D_{\delta}=\left|C_{3} \cdot W_{\delta}-W\right| \\
& W_{1}=W_{\alpha}-A_{1} .\left(D_{\alpha}\right), W_{2}=W_{\beta}-A_{2} .\left(D_{\beta}\right), W_{3}=W_{\delta}-A_{3} .\left(D_{\delta}\right)
\end{aligned}
$$

The algorithm of the GWO is reported as follows.

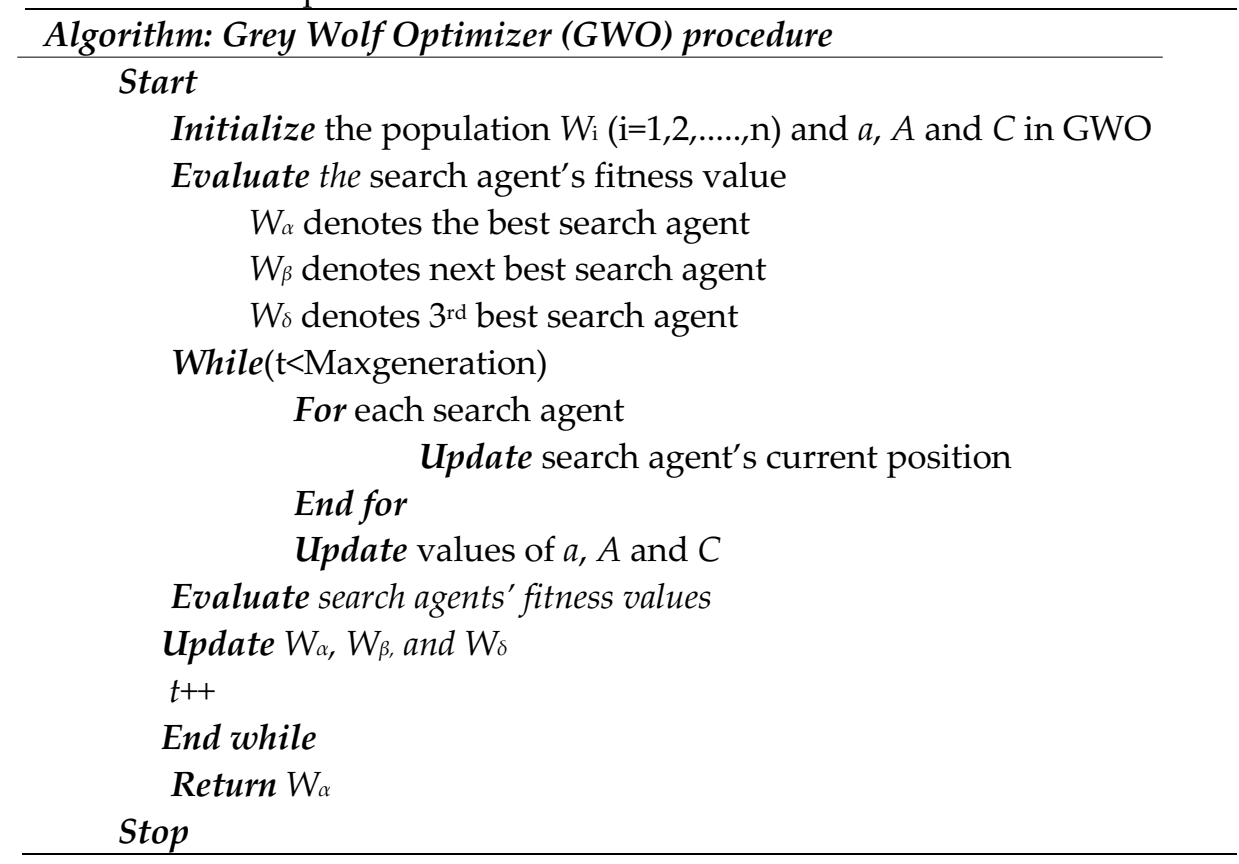

In this work, the fitness function is the correlation coefficient which is used as the objective function. The correlation coefficient is a good metric of understanding the amount of difference between two images to determine the amount of similarity between the original and registered image [27]. The main aim of the proposed work is to increase the registration accuracy, i.e. increase the image quality [29, 43], so that the registered image is almost identical to the target image. Henceforth, the correlation coefficient was chosen 
as the objective function in this current work. Accordingly, the proposed algorithm-based Wang's demons registration is given in Algorithm 2.

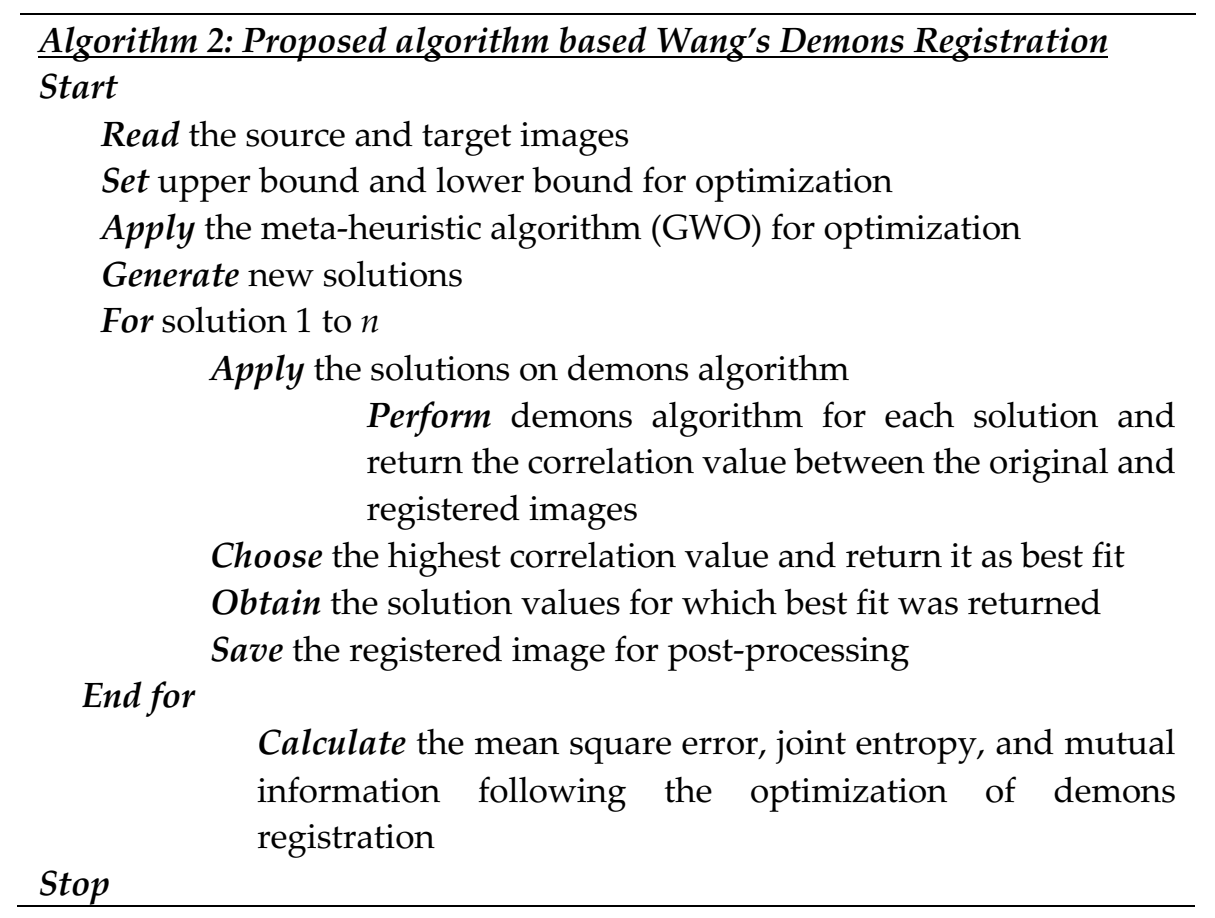

\section{Proposed Method}

As previously discussed in section 3, the objective function of non-rigid registration [42, 43] must include the alignment field's similarity measurement. The present optimizes the parameters of Wang's demons [61], where it is faster and has less error in demons deformation during the registration process compared to the Wang's demons [61], and the Thirion's demons [30]. Hence, Wang's demons is applied in this current work for the image registration framework to determine the optimal value of the alpha noise constant and Gaussian smoothening filter. Typically, the default window size in Thirion's [30] demons as well as Wang's demons [61] is 60×60, and the sigma value is 10 . The block diagram of the proposed method is illustrated in Figure 1. 


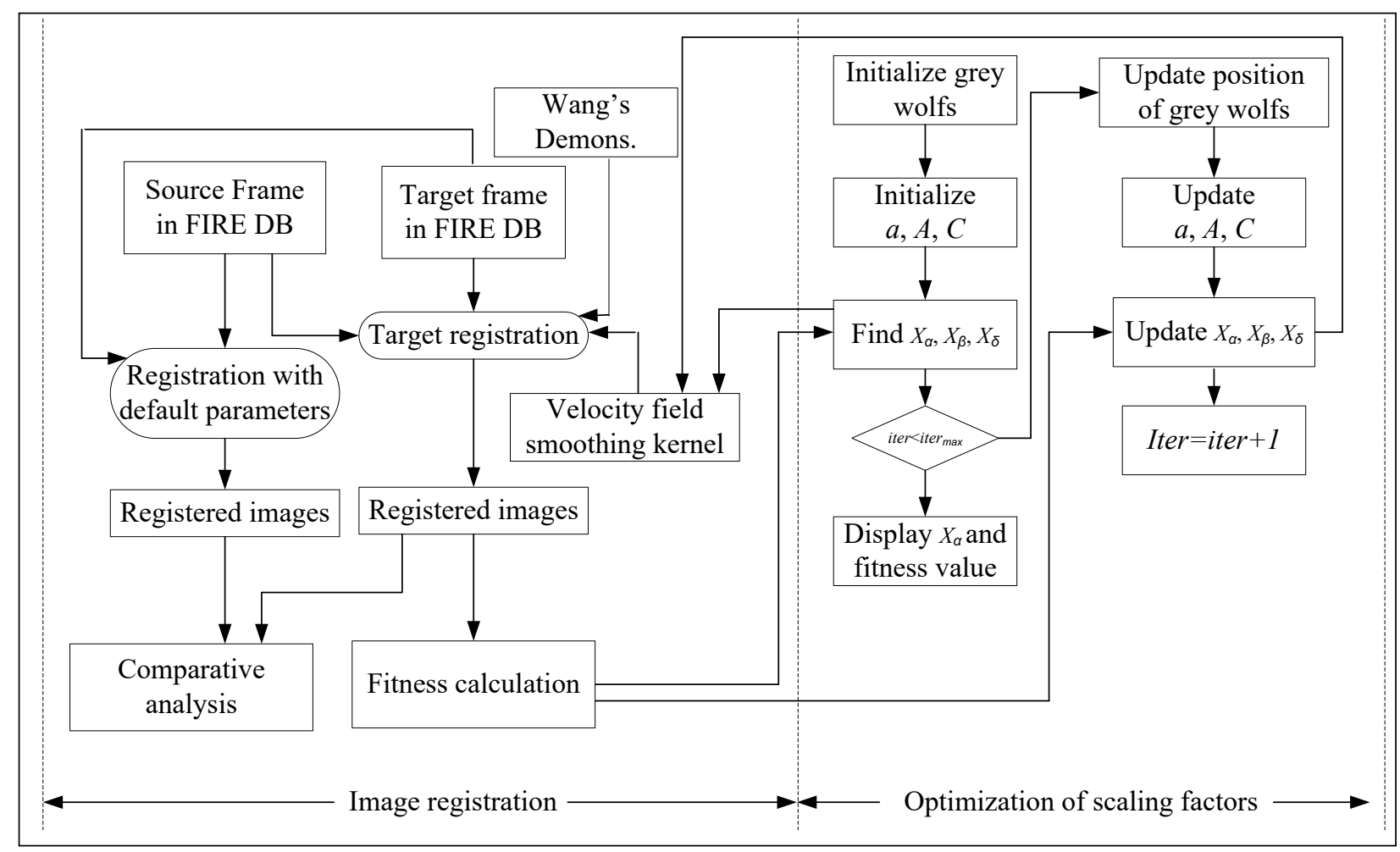

Figure 1. Block diagram of the proposed method.

In the proposed method, the retinal images $[62,63]$ are converted initially into gray-scale images. The image frames [64] were then processed for demons registration. Correlation value $[57,58,59]$ of the original and registered image is considered as the best fitness. The parameters of the demons registration are the scaling factors to be optimized using the GWO. The image with the highest correlation [60] with the original image is chosen to be the optimal registered image and the scaling factors value as well as the correlation value were stored for post-processing. Figure 1 explains the proposed work, where the left side the image registration part is carried out, in which, firstly the source and then target frames are chosen from the database. The registration is done using the default parameters, and optimized parameters that include the Gaussian filter's window size (60×60 pixels), and 2-D sigma value (10). These values are varied from 20 to 100 values for window size and 0-20 for 2-D sigma value during optimization, using grey wolf optimization. The grey wolf optimizer can be observed on the right side of the diagram. It uses the correlation value between the original and registered images to find the best fitness and respective parameter values. After each iteration, values, or position of grey wolves get updated, i.e. the values of the parameters are chosen from the population pool, and the registration is done over and over again until the best fitness is found.

\section{Results and Discussion}

The current work is tested and executed on Intel i3, $2.2 \mathrm{GHz}$ processor-based system with $8 \mathrm{~GB}$ ram, and Windows 10 operating system. The simulation for running the image registration process was done on MatLab R2018a software in which CS, PSO, FA, genetic algorithm (GA), and the proposed GWO has been applied for optimization of the registration framework. The $31^{\text {st }}$ pair from P-set in the FIRE database is chosen for image registration [19]. 


\subsection{Dataset}

The dataset used in this current work was one of the popular datasets available for retinal image processing, named as FIRE-DB [65]. There are 129 retinal images inside the FIRE-DB forming a total of 134 image pairs. These image pairs are further classified and stored according to their different categories. There are three categories: set A, set P, and set $\mathrm{S}$. These images were captured using Nidek AFC-210 fundus camera. The resolution of all images was $2912 \times 2912$ pixels as obtained by the camera. The FOV of images was set at $45^{\circ}$ in $\mathrm{x}$ and $\mathrm{y}$ dimensions. The set of capturing the images was done in Papageorgiou Hospital, Aristotle University of Thessaloniki. 39 patients volunteered for this task. Among these datasets, the same image pair was chosen for the current framework, as discussed in Lan et al. [43]

\subsection{Meta-heuristics based Wang's Demons registration}

Several performance evaluation metrics are measured, where the correlation between two images (X and $Y, X^{\prime}$ and $Y^{\prime}$ are mean of the images) is measured in terms of the correlation coefficient which is given by [39]:

$$
\operatorname{corr}=\frac{\sum_{\mathrm{m}} \sum_{\mathrm{n}}\left(\mathrm{X}_{\mathrm{mn}}-\mathrm{X}^{\prime}\right)\left(\mathrm{Y}_{\mathrm{mn}}-\mathrm{Y}^{\prime}\right)}{\sqrt{\left(\sum_{\mathrm{m}} \sum_{\mathrm{n}}\left(\mathrm{X}_{\mathrm{mn}}-\mathrm{X}^{\prime}\right)^{2}\right)\left(\sum_{\mathrm{m}} \sum_{\mathrm{n}}\left(\mathrm{Y}_{\mathrm{mn}}-\mathrm{Y}^{\prime}\right)^{2}\right)}}
$$

In the present work, several optimization algorithms, including FA, CS, PSO, and GA, are applied to optimize Wang's demons registration parameters for comparative study with the proposed GWO based registration.

\subsubsection{Grey-wolf optimization-based Wang's Demons registration}

Table 2 reported the $k_{1}, k_{2}$ and $k_{3}$ values for Grey Wolf Optimization algorithm-based demons registration framework. The population has been kept fixed at 15 , while iterations are increased by 5 , from $5^{\text {th }}$ generation to $30^{\text {th }}$.

Table 2. Grey Wolf Optimization algorithm-based Wang's Demons registration

\begin{tabular}{cccccll}
\hline & $\mathbf{5} \times \mathbf{1 5}$ & $\mathbf{1 0} \times \mathbf{1 5}$ & $\mathbf{1 5} \times \mathbf{1 5}$ & $\mathbf{2 0} \times \mathbf{1 5}$ & $\mathbf{2 5} \times \mathbf{1 5}$ & $\mathbf{3 0} \times 15$ \\
\hline Correlation & 0.9968 & 0.9969 & 0.9970 & 0.9977 & 0.9977 & 0.9977 \\
\hline $\begin{array}{c}\text { Time for } \\
\text { Optimization }\end{array}$ & 24153.56 & 47175.47 & 70458.02 & 100752.23 & 138726.54 & 174113.1 \\
\hline$k_{1}, k_{2}, k_{3}$ & $89,60,18$ & $97,79,18$ & $98,98,20$ & $99,99,20$ & $99,99,20$ & $99,99,20$ \\
\hline
\end{tabular}

The observation shows that it took 20 iterations to reach convergence and the best fitness value was 0.9977 which was the correlation value between the original and registered image that was achieved by the Gaussian filter value of 99x99 and sigma value of 20. The images involved and the resultant image is shown below. 

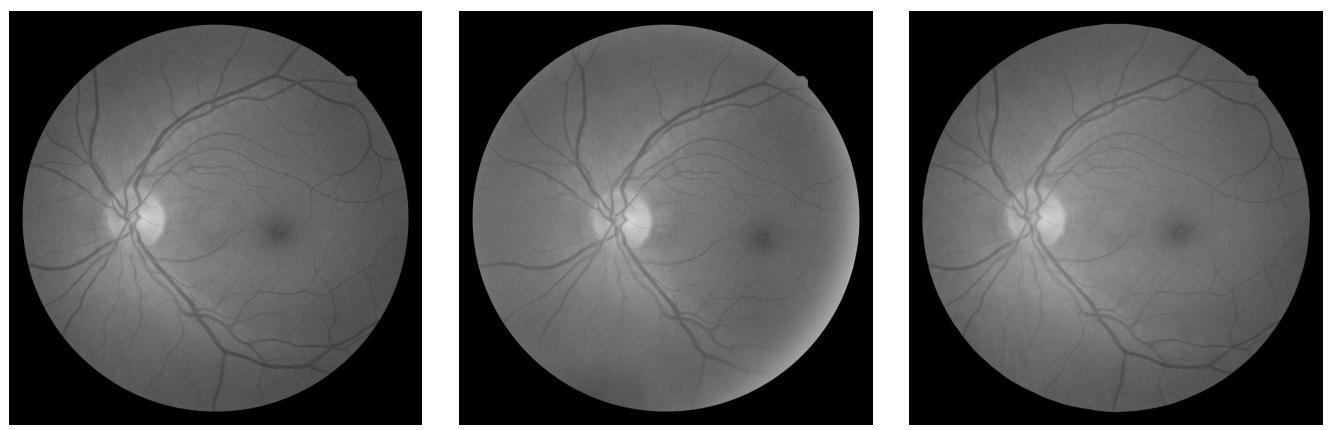

Figure 2. Moving (A), fixed(B) and registered (C) image in Grey Wolf Optimization-based demons registration

\subsubsection{Firefly algorithm-based Wang's Demons registration}

The FA is used to optimize three parameters namely, namely $k_{1}, k_{2}$ and $k_{3}$ for the demons registration framework. Figure 3 includes a sample of the moving, fixed, and registered image using the firefly algorithm based demons registration.
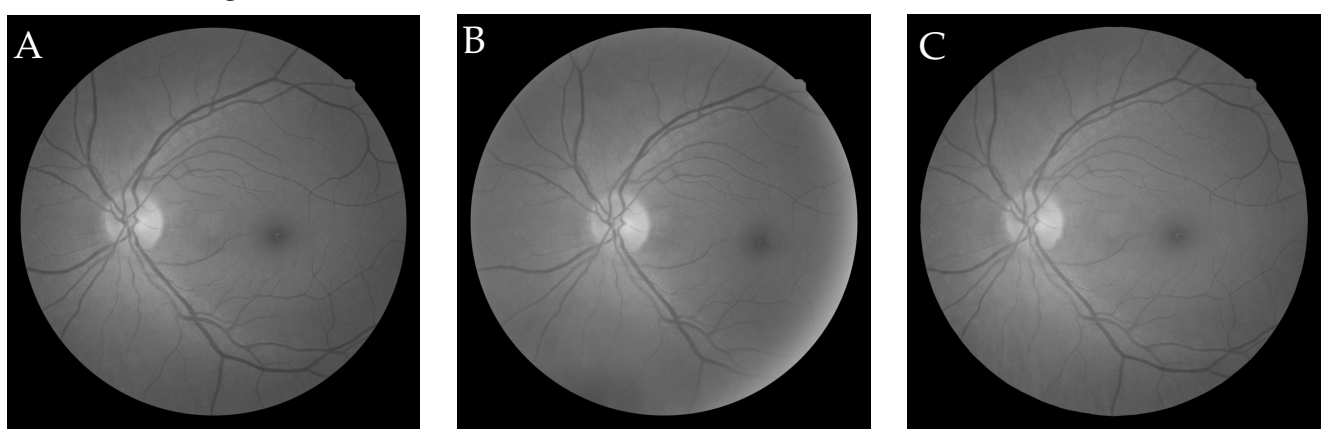

Figure 3. Moving, fixed and registered image in firefly algorithm based demons registration, where (A) reference retinal image, (B) original image, and (C) registered image using FA based Wang's Demons registration

Also, Table 3 reported the evaluation metrics' value of the used FA-based Wang's demons registration, the required optimization time, and the optimal valued of the optimized parameters of Wang's demons registration method.

Table 3. Firefly algorithm-based Wang's Demons registration

\begin{tabular}{ccccccc}
\hline & $\mathbf{5} \times \mathbf{1 5}$ & $\mathbf{1 0} \times \mathbf{1 5}$ & $\mathbf{1 5} \times \mathbf{1 5}$ & $\mathbf{2 0} \times \mathbf{1 5}$ & $\mathbf{2 5} \mathbf{\times 1 5}$ & $\mathbf{3 0 \times 1 5}$ \\
\hline Correlation & 0.9766 & 0.9870 & 0.9969 & 0.9970 & 0.9970 & 0.9970 \\
\hline $\begin{array}{c}\text { Time for } \\
\text { Optimization }\end{array}$ & 25104.92 & 58984.51 & 84369.26 & 132714.22 & 165263.5 & 210714.76 \\
\hline $\boldsymbol{k}_{\mathbf{1}}, \boldsymbol{k}_{\mathbf{2}}, \boldsymbol{k}_{\mathbf{3}}$ & $66,71,19$ & $87,50,18$ & $96,81,20$ & $98,98,20$ & $98,98,20$ & $98,98,20$ \\
\hline
\end{tabular}


The observation shows that it took 20 iterations for the FA to reach convergence and the best fitness value was 0.9970 that was the correlation value between original and registered image.

\subsubsection{Cuckoo search algorithm-based Wang's Demons registration}

Table 4 reported the $k_{1}, k_{2}$ and $k_{3}$ values for the Cuckoo search algorithm-based demons registration framework. The population has been kept fixed at 15 , while iterations are increased by 5 , from $5^{\text {th }}$ generation to $30^{\text {th }}$.

Table 4: Cuckoo search algorithm-based Wang's Demons registration

\begin{tabular}{lcccccc}
\hline & $\mathbf{5} \times \mathbf{~ 1 5}$ & $\mathbf{1 0} \times \mathbf{~ 1 5}$ & $\mathbf{1 5} \times \mathbf{~ 1 5}$ & $\mathbf{2 0} \times \mathbf{1 5}$ & $\mathbf{2 5} \times 15$ & $\mathbf{3 0} \mathbf{x 1 5}$ \\
\hline Correlation & 0.9965 & 0.9977 & 0.9977 & 0.9977 & 0.9977 & 0.9977 \\
\hline $\begin{array}{c}\text { Time for } \\
\text { Optimization }\end{array}$ & 24820.26 & 53485.82 & 81148.52 & 121591.49 & 156132.41 & 211056.74 \\
\hline$k_{1}, k_{2}, k_{3}$ & $67,92,18$ & $99,99,20$ & $99,99,20$ & $99,99,20$ & $99,99,20$ & $99,99,20$ \\
\hline
\end{tabular}

The observation shows that it took 20 iterations to reach convergence and the best fitness value was 0.9970 which was the correlation value between the original and registered image that was achieved by the Gaussian filter value of 99x99 and sigma value of 20. Clearly, the results are better than the previous two methods (FA and GWO). The images involved and the resultant image are shown below.
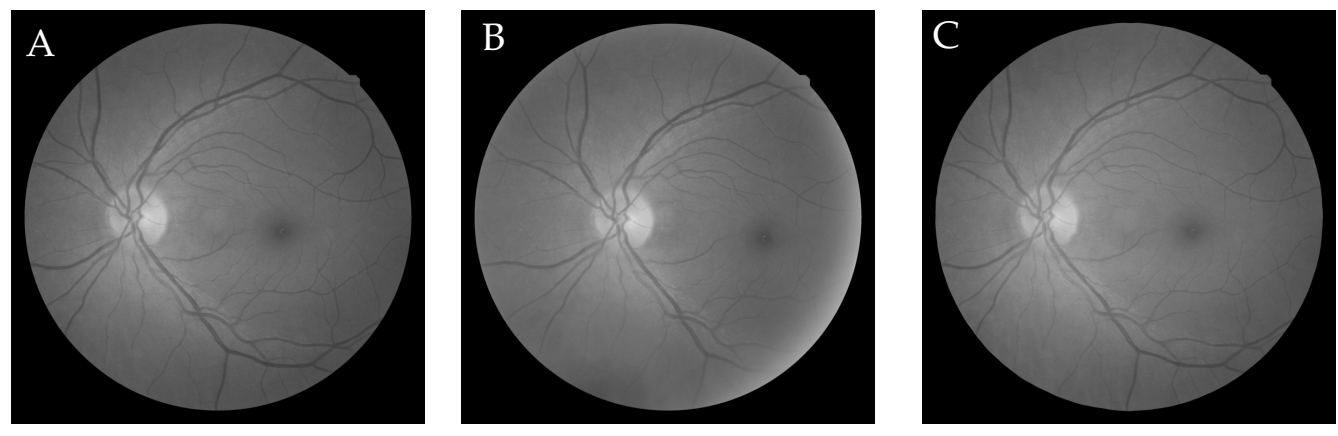

Figure 4. Moving, fixed and registered image in Cuckoo search algorithm-based demons registration

\subsubsection{Genetic algorithm-based Wang's Demons registration}

The same observation was done using the Genetic algorithm as well and the results are shown in Table 5. Same as the previous three methods, here also the population has been kept fixed at 15, while iterations are increased by 5 , from $5^{\text {th }}$ generation to $30^{\text {th }}$.

Table 5. Genetic algorithm based Wang's Demons

\begin{tabular}{ccccccc}
\hline & $\mathbf{5} \times \mathbf{1 5}$ & $\mathbf{1 0} \times \mathbf{~ 1 5}$ & $\mathbf{1 5} \times \mathbf{1 5}$ & $\mathbf{2 0} \times \mathbf{1 5}$ & $\mathbf{2 5} \times \mathbf{~ 1 5}$ & $\mathbf{3 0} \times \mathbf{~ 1 5}$ \\
\hline Correlation & 0.9157 & 0.9265 & 0.9266 & 0.9266 & 0.9266 & 0.9266 \\
\hline $\begin{array}{c}\text { Time for } \\
\text { Optimization }\end{array}$ & 4909.18 & 10104.6 & 14314.98 & 19729.19 & 23934.43 & 29177.21 \\
\hline$k_{1}, k_{2}, k_{3}$ & $74,74,15$ & $94,94,19$ & $96,96,19$ & $96,96,19$ & $96,96,19$ & $96,96,19$ \\
\hline
\end{tabular}

The original image, registered image, and reference image involved in the genetic algorithm based framework for Wang's demons registration are illustrated below. 

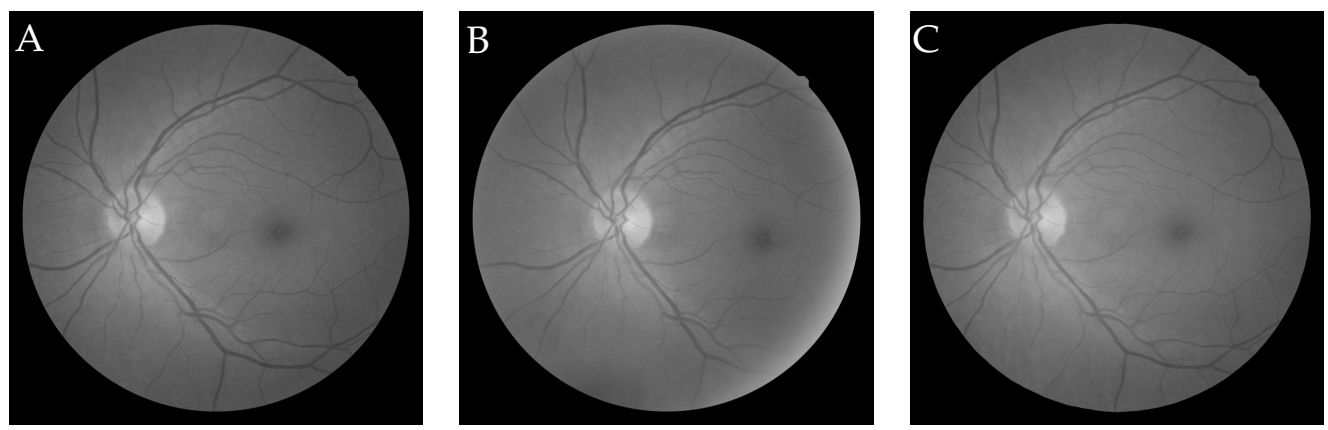

Figure 6. Moving (A), fixed (B) and registered (C) image in Genetic Algorithm based demons registration

\subsubsection{Particle swarm optimization algorithm-based Wang's Demons registration}

To keep the analysis simple, the same process was continued for particle swarm optimization as well and the results are shown in Table 6. Same as the previous three methods, here also the population has been kept fixed at 15 , while iterations are increased by 5 , from $5^{\text {th }}$ generation to $30^{\text {th }}$.

Table 6. Particle swarm optimization algorithm based Wang's Demons

\begin{tabular}{ccccccc}
\hline & $\mathbf{5} \times \mathbf{1 5}$ & $\mathbf{1 0} \times \mathbf{1 5}$ & $\mathbf{1 5} \times \mathbf{1 5}$ & $\mathbf{2 0} \times \mathbf{1 5}$ & $\mathbf{2 5} \times 15$ & $\mathbf{3 0} \times 15$ \\
\hline Correlation & 0.9657 & 0.9960 & 0.9970 & 0.9970 & 0.9970 & 0.9970 \\
\hline $\begin{array}{c}\text { Time for } \\
\text { Optimization }\end{array}$ & 56841.91 & 104131.62 & 143152.31 & 198331.62 & 224136.44 & 297528.52 \\
\hline$k_{1}, k_{2}, k_{3}$ & $64,72,18$ & $92,76,19$ & $98,98,20$ & $98,98,20$ & $98,98,20$ & $98,98,20$ \\
\hline
\end{tabular}

The results are poorer compared to the previous three methods, as the framework took longer time to optimize as well as, the resultant images are no better than the firefly algorithm or grey-wolf optimizationbased algorithm's results. The original images involved and the resultant image are shown below.
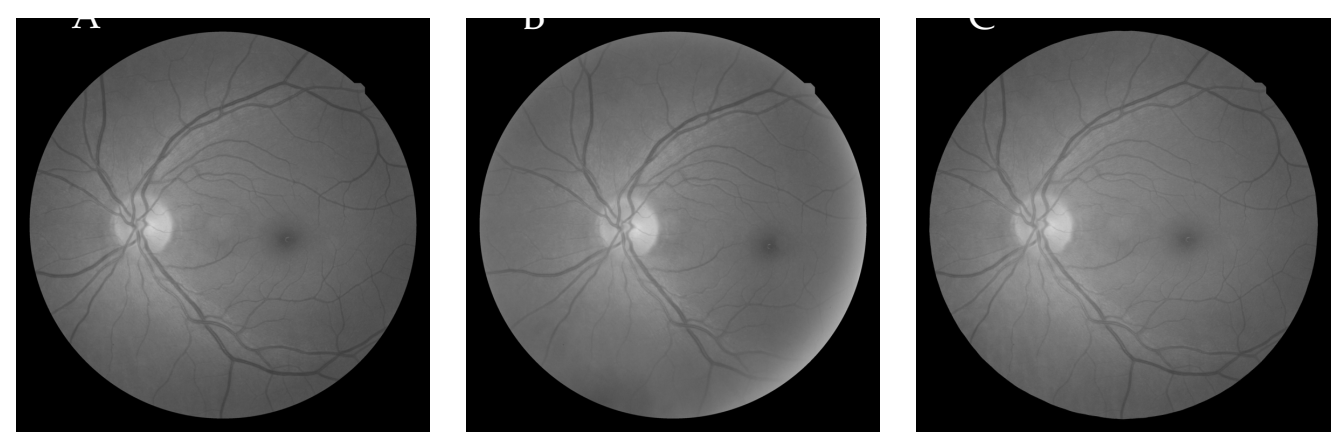

Figure 7. Moving (A), fixed (B) and registered (C) image in Particle Swarm Optimization based demons registration

As previously discussed, the fundus images that were used were taken from fire-DB, and a pair of fundus images were chosen from the database, that was used in Lan et al.'s work [43].

The values of the parameters that were optimized were the window size of the gaussian low pass filter, with a range varying from 20-100 and sigma range varying from 0-20 as reported in Table 7 . 
Table 7. The parameters to be optimized

\begin{tabular}{|c|c|c|}
\hline Total number of iterations & 120 & \\
\hline \multirow{2}{*}{ Velocity field smoothing kernel } & 2-D filter & Sigma \\
\hline & $\begin{array}{l}\text { Gaussian low pass } \\
\text { filter } \\
\text { of window size } 60 \times 60\end{array}$ & 10 pixels \\
\hline Alpha (noise) constant & $2.5 \mathrm{~dB}$ & \\
\hline
\end{tabular}

6.2.6. Comparative study in terms of the time complexity and the fitness values using Wang's Demons registration

The time complexity of the used meta-heuristic frameworks-based demons registration is analyzed as illustrated in Figure 7.

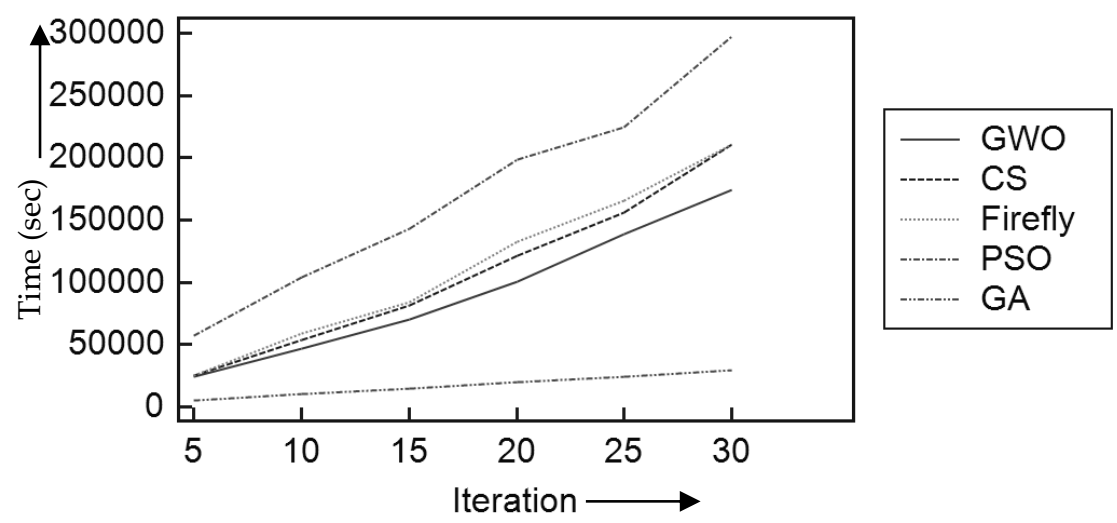

Figure 7. Comparative study of time complexity in meta-heuristic algorithms-based demons registration

Figure 7 revealed that GA achieved the fastest convergence during the optimization process. However, the GWO performed superior results in terms of the time complexity compared to the FA-, GA- and PSObased registration methods. Moreover, Figure 8 demonstrates the box plot of the comparative study in terms of the achieved fitness values using the different meta-heuristic algorithms-based demons registration.

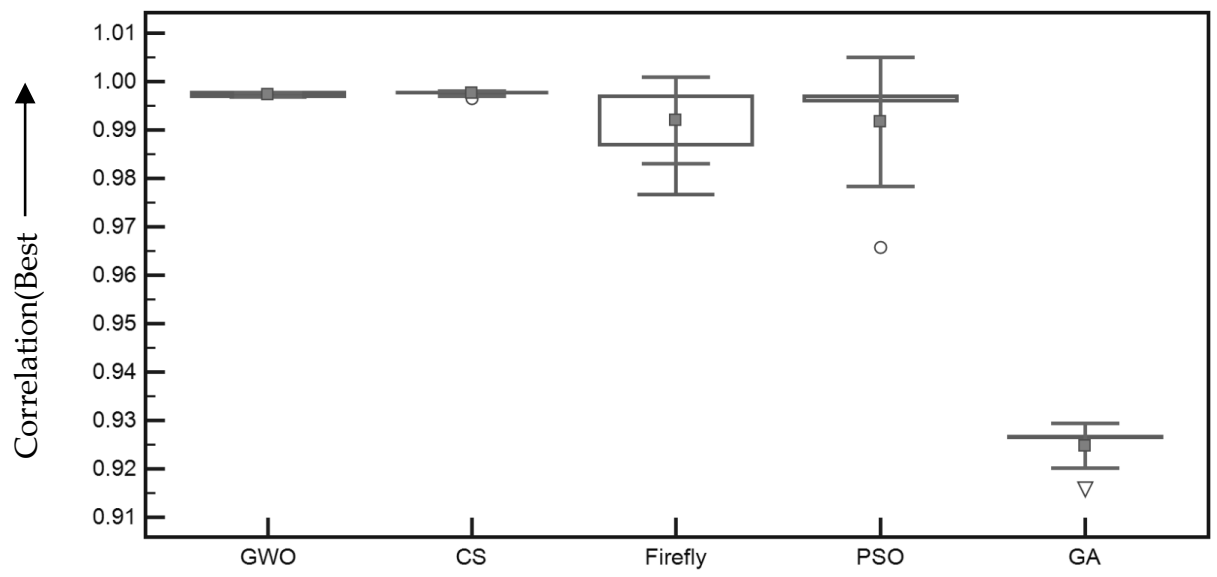


Figure 8. Comparative study of fitness values in meta-heuristic algorithms based demons registration

Figure 8 reported that the best/highest fitness (correlation) value is achieved using the PSO- based registration. However, the GWO provided the most stable with less variation in the fitness values. To illustrate the convergence speed, Figs. 9 and 10 are plotted to illustrate the comparative study of fitness values in the applied meta-heuristic algorithms-based demons registration using the line plot.

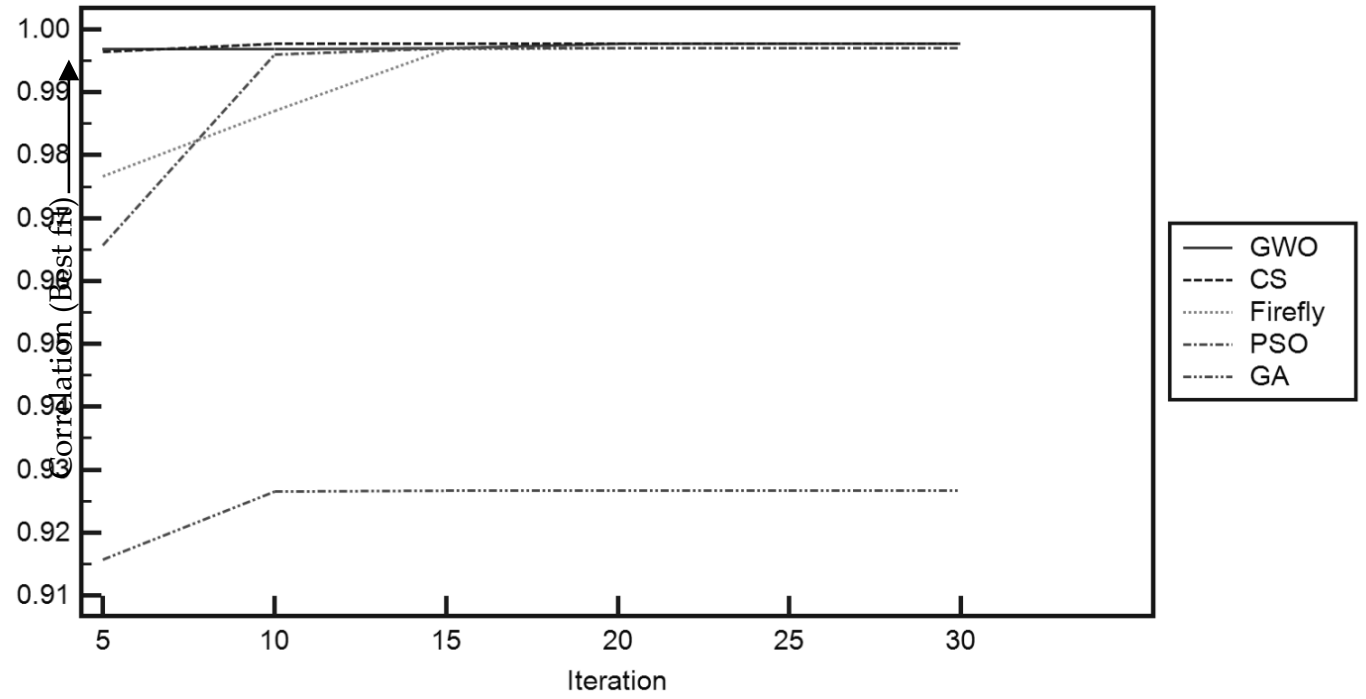

Figure 9. Comparative study of fitness values in meta-heuristic algorithms-based demons registration

Figures 9 and 10 depicted that the FA took a greater number of iterations for complete convergence, while both the GWO and the GA provided the best fitness value with the least number of iterations (about 5 iterations). 


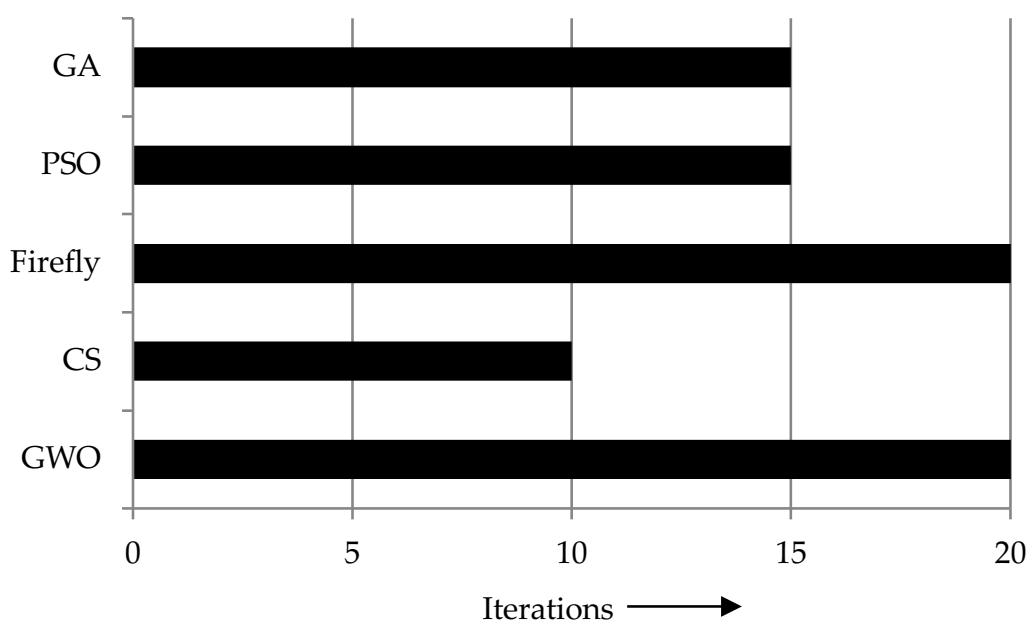

Figure 10. Comparative study of required iterations in meta-heuristic algorithms-based demons registration

\subsection{Comparative study of the GWO-based different Demons registration}

The following study is carried out on different demons algorithm in order to establish the superior demons algorithm that worked well with grey wolf optimization. The first algorithm to undergo the procedure was Wang's demons. The results are shown in Table 8 which reports the best fitness value was achieved after the $15^{\text {th }}$ iteration.

Table 8. Grey Wolf Optimization algorithm-based Wang's Demons registration

\begin{tabular}{ccccccc}
\hline & $\mathbf{5} \times \mathbf{~ 1 5}$ & $\mathbf{1 0} \times \mathbf{~ 1 5}$ & $\mathbf{1 5} \mathbf{1 5}$ & $\mathbf{2 0} \mathbf{1 5}$ & $\mathbf{2 5 \times 1 5}$ & $\mathbf{3 0 \times 1 5}$ \\
Correlation & 0.9968 & 0.9969 & 0.9970 & 0.9977 & 0.9977 & 0.9977 \\
Time for Optimization & 24153.56 & 47175.47 & 70458.02 & 100752.23 & 138726.54 & 174113.1 \\
$k_{1}, k_{2}, k_{3}$ & $89,60,18$ & $97,79,18$ & $98,98,20$ & $99,99,20$ & $99,99,20$ & $99,99,20$ \\
\hline
\end{tabular}

The obtained images are shown in Figure 11. The reference, original, and register image shows the quality of the registered image was quite higher.
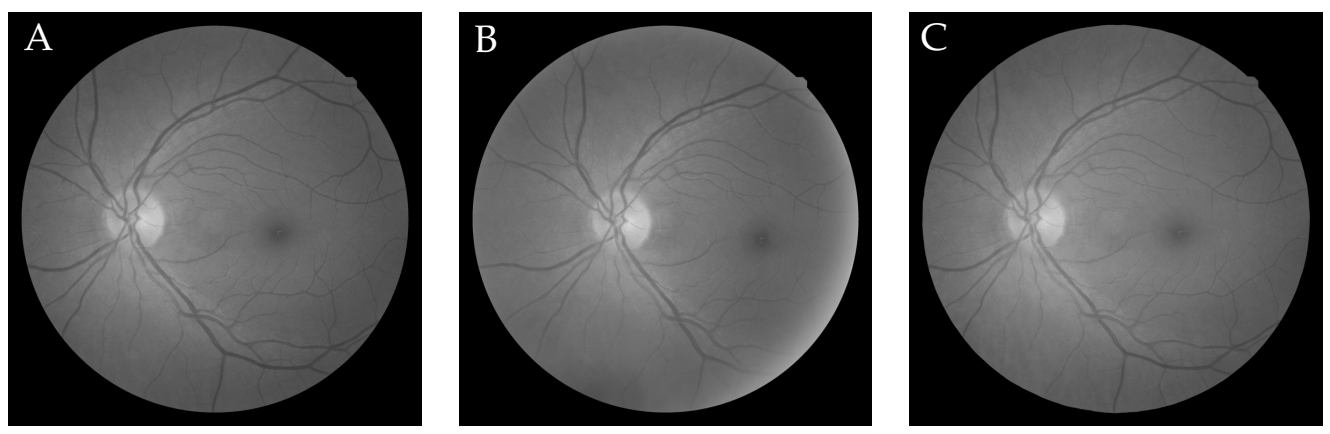

Figure 11. Moving (A), fixed (B) and registered image (C) in Grey Wolf Optimization-based Wang's demons registration

The next registration method to undergo the procedure was Tang's demons. The results are shown in Table 8 which reports, the best fitness value was achieved after the $15^{\text {th }}$ iteration. But this time the correlation was lower than Wang's demons. 
Table 7. Grey Wolf Optimization algorithm-based Tang's Demons registration

\begin{tabular}{ccccccc}
\hline & $\mathbf{5} \times \mathbf{1 5}$ & $\mathbf{1 0} \times \mathbf{1 5}$ & $\mathbf{1 5} \times \mathbf{1 5}$ & $\mathbf{2 0 \times 1 5}$ & $\mathbf{2 5} \times 15$ & $\mathbf{3 0 \times 1 5}$ \\
\hline Correlation & 0.9967 & 0.9969 & 0.9967 & 0.9970 & 0.9970 & 0.9970 \\
\hline $\begin{array}{c}\text { Time for } \\
\text { Optimization }\end{array}$ & 27703.23 & 48149.94 & 71915.53 & 101793.91 & 146753.1 & 175665.3 \\
\hline$k_{1}, k_{2}, k_{3}$ & $74,98,18$ & $79,99,20$ & $80,91,18$ & $98,98,20$ & $98,98,20$ & $98,98,20$ \\
\hline
\end{tabular}

The obtained images are shown in Figure 12. The reference, original, and register image shows the quality of the registered image was quite lower than Wang's demons.
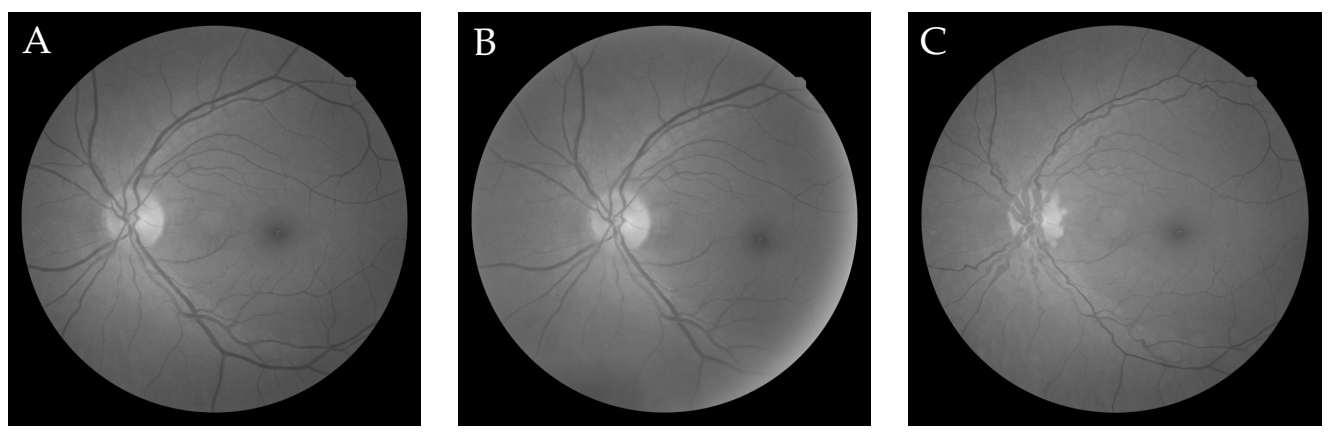

Figure 12. Moving (A), fixed (B) and registered (C) image in Grey Wolf Optimization-based Tang's demons registration

The final registration method to undergo the procedure was Thirion's demons. The results are shown in Table 9 which reports, the best fitness value was achieved after the $15^{\text {th }}$ iteration. But this time also the correlation was lower than Wang's demons.

Table 9. Grey Wolf Optimization algorithm-based Thirion's Demons registration

\begin{tabular}{ccccccc}
\hline & $\mathbf{5 \times 1 5}$ & $\mathbf{1 0 \times 1 5}$ & $\mathbf{1 5 \times 1 5}$ & $\mathbf{2 0 \times 1 5}$ & $\mathbf{2 5 \times 1 5}$ & $\mathbf{3 0 \times 1 5}$ \\
\hline Correlation & 0.9970 & 0.9969 & 0.9969 & 0.9970 & 0.9970 & 0.9970 \\
\hline $\begin{array}{c}\text { Time for } \\
\text { Optimization }\end{array}$ & 23927.88 & 50362.82 & 73978.46 & 103359.34 & 149332.8 & 175360.6 \\
\hline$k_{1}, k_{2}, k_{3}$ & $96,94,19$ & $53,99,19$ & $68,98,20$ & $98,98,20$ & $98,98,20$ & $98,98,20$ \\
\hline
\end{tabular}

The obtained images are shown in Figure 13. The reference, original and register image shows the quality of the registered image was also lower than Wang's demons.
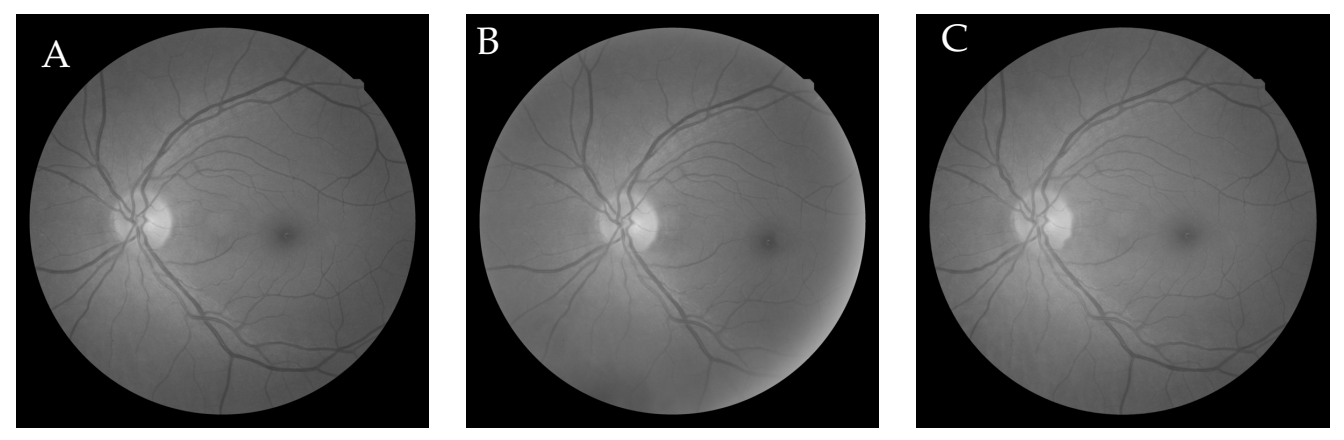

Figure 13. Moving (A), fixed (B) and registered (C) image in Grey Wolf Optimization based Thirion's demons registration 
A comparative analysis of time complexity has been observed in Figure 14 which shows that Wang's demons took lesser time to find the optimal solution to the registration problem compared to the other two methods.

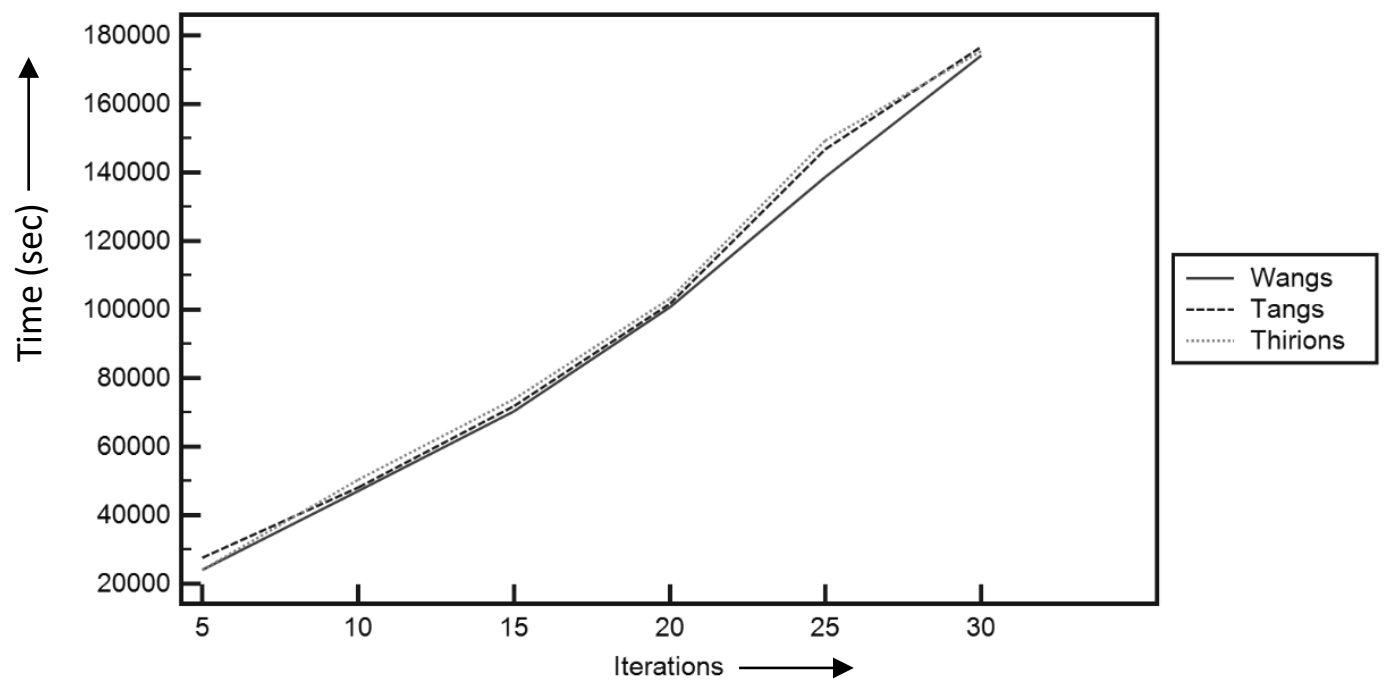

Figure 14. Comparative study of time complexity of different Grey wolf optimization-based demons algorithm

Similarly, a comparative study of best fitness or correlation values has been observed in Figure 15. It shows, Wang's demons took lesser iterations as well to find the optimal solution to the registration problem compared to the other two methods. The optimal solution itself was better than the other two methods.

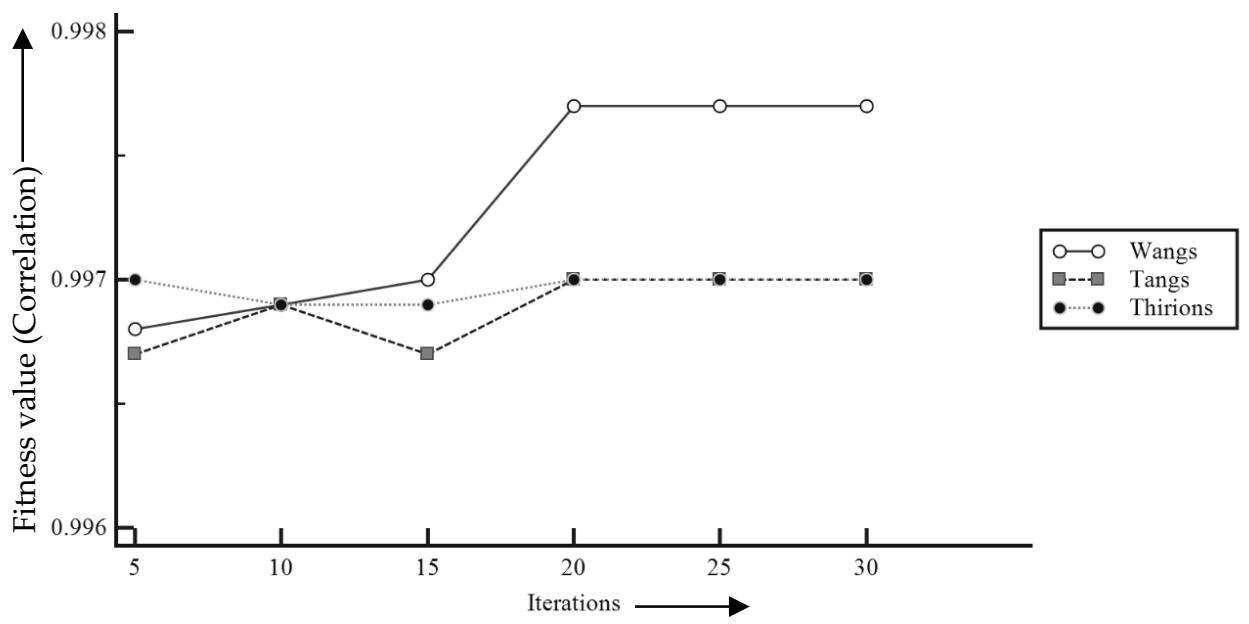

Figure 15. Comparative study of best fitness of different Grey wolf optimization-based demons

Finally, Table 10 reports various image metric's quantity while exploring grey-wolf optimizationbased, different demons algorithms. The mean square error (MSE) refers to the total squared error between the registered and the original image, which is expressed as follows [43]:

$$
M S E=\frac{1}{M N} \sum_{y=1}^{M} \sum_{x=1}^{N}[I(x, y)-J(x, y)]^{2}
$$


The joint entropy of two images $\mathrm{X}$ and $\mathrm{Y}$ is calculated using the following expression [43]:

$$
\operatorname{MJE}(X, Y)=-\sum_{x \in X} \sum_{y \in Y} P(x, y) \log _{2}[P(x, y)]
$$

In addition, the mutual information of the image $X$ and $Y$ can be defined as [43]:

$$
N M I=\operatorname{entropy}(X)+\operatorname{entropy}(Y)-M J E(X, Y)
$$

The mean square error, mean joint entropy, and net mutual information values are observed for different demons framework that was based on grey-wolf optimization. Table 8 reports the results of MSE, MJE, NMI, and correlation values. The mean square error was significantly low in Wang's demons $\left(8.3585 \times 10^{-5}\right)$ registration compared to other two as the higher correlation value $(0.9977)$ of Wang's supports the claim of obtaining better quality images as a result of grey-wolf optimized Wang's demons registration, than Tang's registration and Thirion's registration's framework.

Table 10.Comparative study of Grey Wolf Optimization algorithm-based Demons registration

\begin{tabular}{cccc}
\hline Optimized values & Wang's Demons & Tang's Demons & Thirion's demons \\
\hline MSE & $8.3585 \times 10^{-5}$ & $2.7314 \times 10^{-4}$ & $2.6885 \times 10^{-4}$ \\
\hline MJE & 5.3007 & 5.3464 & 5.3456 \\
\hline NMI & 0.4920 & 0.4493 & 0.4500 \\
\hline Correlation & 0.9977 & 0.9970 & 0.9970 \\
\hline
\end{tabular}

Figure 16 showed the overlapped images of the original image and obtained images from three different demons registration frameworks of Wang's, Tang's, and Thirion's demons, based on the greywolf optimization.

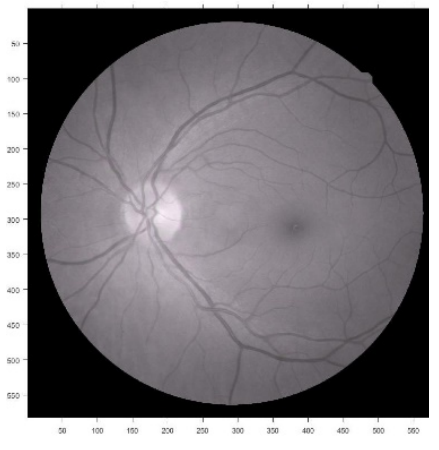

Wangs

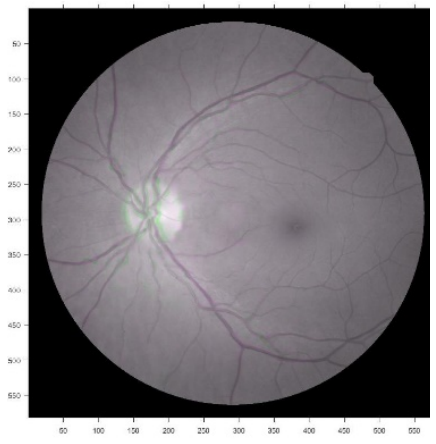

Tangs

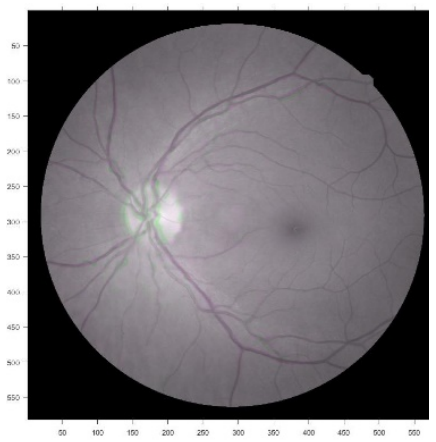

Thiron's

Figure 16. Comparative study of overlapped areas of original and registered images in different Grey wolf optimization-based demons

From the observation in Figure 16 and Table 8, it can be said that Grey wolf optimization-based Wang's demons performed better in terms of obtaining quality image registration. Although the time complexity study has shown that Thirion's demons managed to achieve the convergence earlier than Wang's and Tang's demons registration based on the GWO algorithm. GWO based Wang's demons performed faster during registration and optimization than the other two methods, making it superior among the three demons registration. 
19 of 24

\section{Discussion}

Table 11 provides a comparison for 8 of the existing techniques that experimented on retinal image registration algorithms, previously. Among them, in 2005, Chanwimaluang used are based registration on retinal images obtained from area NIH. They used Feature-based [10] image registration and affine registration as the benchmark and reported $95.1 \%$ registration accuracy compared to $94.7 \%$ and $90.4 \%$ accuracy of the other two image registration techniques respectively. In 2012, Gharabghi proposed retinal image registration using a geometrical [23] feature. In this work, the DRIVE database reported 96\% accuracy. Later in 2016, Parekar et al. [62] used feature matching registration on retinal images, which reported a mean error of 1.785, and a standard deviation of 0.974 , compared to Generalized Dual-Bootstrap Iterative closest Point's (GDB-ICP) 3.505 mean error and 2.789 standard deviations. As discussed previously, Hernandez-Matas et al. [22] introduced the rigid registration on retinal images in 2017 using swarm intelligence to solve registration errors generated during the rigid registration process. The results reported a mean error of 0.43 , compared to 0.68 of their previous method of Hernandez-Matas et al. 2015 and 7.20 of the RANSAC method. They also reported an improvement of $57.75 \%$ on registration accuracy while comparing it with the RANSAC method and 37.93\% while comparing it with Hernandez-Matas et al., 2015. Ramli et al. [21] discussed the effect of feature-based registration, in which he used the D-SADDLE feature on retinal image registration. They measured registration accuracy based on the amount of retinal image pair, that was registered successfully. They reported a registration accuracy of $43 \%$ but also accepted that the method would fail if the original and reference frame had small overlapping areas. In 2017, Hang et al. proposed a SIFT feature based retinal image registration. In this work, they used bifurcation [63] points as a feature for registering images. They used matching points to measure the accuracy of registration, and the best they could get was 38\% accuracy. Like the previous method, their method also failed for registering images having a small overlap area. Li et al. introduced retinal image registration [12], that was featurebased, but the novelty of this method was, it tried to address the image modality issues. They used the Dice coefficient to measure the registration accuracy. In this work, the found the dice coefficient value to be $0.74 \pm 0.08$ than $0.62 \pm 0.09$ descriptor matching registration and $0.60 \pm 0.17$ of Miri et al.'s work. Later in 2018, Tang et al. introduced a novel non-rigid [64] point matching registration. They tested the success rate of their method and compared it to UR-SIFT-PIIFD which is a popular approach in multimodel retinal image registration. The success rates are $92.44 \%$ and $90.21 \%$, respectively.

Table 11. Summary of studies that presented various types of retinal image registration and their corresponding findings and limitations

\begin{tabular}{|l|l|l|l|l|l|l|}
\hline $\begin{array}{l}\text { Sl. } \\
\text { No. }\end{array}$ & Year & $\begin{array}{l}\text { Technique } \\
\text { Used }\end{array}$ & $\begin{array}{l}\text { Database } \\
\text { used }\end{array}$ & $\begin{array}{l}\text { Size } \\
\text { of } \\
\text { data }\end{array}$ & Complexity & Limitations \\
\hline 1 & $\begin{array}{l}\text { Chanwi } \\
\text { maluang } \\
2005 \\
{[10]}\end{array}$ & $\begin{array}{l}\text { Area-based } \\
\text { registration } \\
\text { and Feature- } \\
\text { based } \\
\text { registration, } \\
\text { Affine } \\
\text { registration }\end{array}$ & NIH & 1008 & Accuracy of 95.1\% & $\begin{array}{l}\text { The time complexity of } \\
\text { processing not } \\
\text { discussed/addressed }\end{array}$ \\
\hline 2 & $\begin{array}{l}\text { Gharaba } \\
\text { ghi } 2012 \\
{[23]}\end{array}$ & $\begin{array}{l}\text { Geometrical } \\
\text { feature- } \\
\text { based } \\
\text { registration }\end{array}$ & DRIVE & 20 & Accuracy of $96 \%$ & $\begin{array}{l}\text { Multimodal registration not } \\
\text { taken into account }\end{array}$ \\
\hline
\end{tabular}




\begin{tabular}{|c|c|c|c|c|c|c|}
\hline 3 & $\begin{array}{l}\text { Parekar } \\
\text { et al. } \\
2016 \\
{[62]}\end{array}$ & $\begin{array}{l}\text { Feature } \\
\text { matching } \\
\text { registration }\end{array}$ & DRIVE & 12 & $\begin{array}{l}\text { Mean error }(2.789) \\
\text { and std dev }(0.974)\end{array}$ & $\begin{array}{l}\text { The concept was based on } \\
\text { similarity transformation }\end{array}$ \\
\hline 4 & $\begin{array}{l}\text { Hernand } \\
\text { ez-Matas } \\
\text { et al. } \\
2017 \\
{[22]}\end{array}$ & $\begin{array}{l}\text { Rigid } \\
\text { registration } \\
\text { with PSO }\end{array}$ & FIRE & 116 & $\begin{array}{l}\text { Registration error } \\
\text { measured ( } 28 \% \\
\text { enhanced) }\end{array}$ & $\begin{array}{l}\text { A comparative study of other } \\
\text { optimization method was } \\
\text { missing. } \\
\text { Discussion about the inclusion of } \\
\text { non-rigid registration was } \\
\text { missing. }\end{array}$ \\
\hline 5 & $\begin{array}{l}\text { Ramli et } \\
\text { al. } 2017 \\
{[21]}\end{array}$ & $\begin{array}{l}\text { Feature- } \\
\text { based } \\
\text { Registration } \\
\text { (D-Saddle } \\
\text { feature) }\end{array}$ & FIRE & 134 & $\begin{array}{l}\text { Registration accuracy } \\
\text { was measured ( } 43 \%)\end{array}$ & $\begin{array}{l}\text { Applied on Mono-modal images } \\
\text { only. } \\
\text { The method fails for small } \\
\text { overlapping areas. } \\
\text { Time complexity not addressed }\end{array}$ \\
\hline 6 & $\begin{array}{l}\text { Hang et } \\
\text { al. } 2017 \\
{[63]}\end{array}$ & $\begin{array}{l}\text { SIFT Feature } \\
\text { based } \\
\text { registration }\end{array}$ & FIRE & 134 & 2.52 seconds/frame & $\begin{array}{l}\text { The method fails when } \\
\text { overlapping areas of the two } \\
\text { images are very small. }\end{array}$ \\
\hline 7 & $\begin{array}{l}\text { Li et al. } \\
2018 \\
{[12]}\end{array}$ & $\begin{array}{l}\text { Feature- } \\
\text { based } \\
\text { multimodal } \\
\text { registration }\end{array}$ & $\begin{array}{l}\text { Universit } \\
\text { y Eye } \\
\text { Clinic } \\
\text { Maastric } \\
\text { ht, } \\
\text { Maastric } \\
\text { ht }\end{array}$ & 600 & $\begin{array}{l}\text { Dice coefficient was } \\
\text { measured ( } 22 \% \\
\text { enhancement than the } \\
\text { existing method) }\end{array}$ & $\begin{array}{l}\text { Comparative analysis was weak. } \\
\text { Time complexity not discussed }\end{array}$ \\
\hline 8 & $\begin{array}{l}\text { Tang et } \\
\text { al. } 2018 \\
{[64]}\end{array}$ & $\begin{array}{l}\text { Non-rigid } \\
\text { point } \\
\text { matching } \\
\text { registration }\end{array}$ & $\begin{array}{l}\text { http://im } \\
\text { agebank. } \\
\text { asrs.org/ }\end{array}$ & 200 & $\begin{array}{l}\text { MSE measured ( } 55 \% \\
\text { reduction than } \\
\text { benchmarks) }\end{array}$ & $\begin{array}{l}\text { The feature matching technique } \\
\text { wasn't robust. Time complexity } \\
\text { not discussed }\end{array}$ \\
\hline
\end{tabular}

The current work discusses the enhancement of the image following the optimization using the Greywolf algorithm. The proposed work also used other similar demons registration algorithm to compare the current framework's effectiveness. In addition, the proposed system has been compared with other optimization methods as well, in which it has reported superior results compared to the frameworks based on cuckoo search, firefly algorithm, particle swarm optimization, and genetic algorithm. Finally, in Table 12 the overall improvement as a result of the grey-wolf optimization-based retinal image registration has been reported.

Table 12. Effect of Grey-wolf optimization-based Demons registration

\begin{tabular}{cccc}
\hline & Original parameters & Optimized parameters & Improvements \\
\hline MSE & $3.6971 \times 10^{-4}$ & $8.3585 \times 10^{-5}$ & 77.39173947 (Decrease) \\
\hline MJE & 5.3615 & 5.3007 & 1.134011004 (Decrease) \\
\hline NMI & 0.4408 & 0.4920 & 10.40650407 (Increase) \\
\hline $\begin{array}{c}\text { Correlation } \\
\text { Registration }\end{array}$ & 0.4666 & 0.9977 & 53.2324346 (Increase) \\
\hline
\end{tabular}

Table 12 clearly shows the improvement of image quality following the image registration using greywolf optimized parameters. Additionally, the reduction of mean-square error following registration supports the claim. The time complexity has also been addressed during this process as it is visible that a 
$3.41 \%$ reduction of time has taken place as a result of using optimization. The reported case is for 1 frame at a time, hence optimization in image registration will address the time complexity problems of registration frameworks that use medical video of large scale.

\section{Conclusion}

The current work presented an analysis of grey wolf optimization and its effect on Wang's demons registration. In the previous work of Lan et al. and Chakraborty et al., Wang's demons were already proven superior to the existing methods of Tang's demons and Thirion's demons. Hence, Wang's demon was chosen for the current framework. The outcome of the current work showed a Grey wolf optimizationbased demons registration framework is one of the fastest and quickest to reach convergence among the existing four meta-heuristic algorithm-based approaches. The results have also indicated that cuckoo search and grey-wolf optimization both produced better resultant images in terms of accuracy of demons registration since it had the highest correlation (0.9977) between original and registered images. The current work is still a pilot in terms of nature. Future work may consist of exploring other meta-heuristic algorithms and combining them with different demons registration framework to analyze the prospect of optimization in the domain of image registration. Future work may include a comparison with the rigid registration method in a similar framework. Multimodal registration is not addressed in the current work, so this might be addressed in future works.

\section{References}

1. Hajnal VJ, Hill DLG, Hawkes DJ. Medical Image Registration. Boca Raton: CRC LLC; 2001. [Google Scholar]

2. Pohl C, Van Genderen JL. Multisensor image fusion in remote sensing: concepts, methods and application. Int J Remote Sens. 1998;19:823-854. doi: 10.1080/014311698215748. [CrossRef] [Google Scholar]

3. Daneshvar S, Ghassemian H. MRI and PET image fusion by combining IHS and retina-inspired models. Inform Fusion. 2010;11:114-123. doi: 10.1016/j.inffus.2009.05.003. [CrossRef] [Google Scholar]

4. Patton N, Aslam TM, MacGillivray T, Deary JI, Dhillon B, Eikelboom RH, Yogesan K, Constable IJ. Retinal image analysis: concepts, applications and potential. Prog Retin Eye Res. 2006;25:99-127. doi: 10.1016/j.preteyeres.2005.07.001. [PubMed] [CrossRef] [Google Scholar]

5. Walter T, Massin P, Erginay A, Ordonez R, Jeulin C, Klein JC. Automatic detection of microaneurysms in color fundus images. Med Image Anal. 2007;11:555-566. doi: 10.1016/j.media.2007.05.001. [PubMed] [CrossRef] [Google Scholar]

6. Yan M, Huang K. A region based algorithm for vessel detection in retinal images. Med Image Comput Comput Assist Interv. 2006;1:645-653. [PubMed] [Google Scholar]

7. Zhu YM. Mutual information-based registration of temporal and stereo retinal images using constrained optimization. Comput Meth Prog Bio. 2007;86:210-215. doi: 10.1016/j.cmpb.2007.02.007. [PubMed] [CrossRef] [Google Scholar]

8. Cideciyan AV. Registration of ocular fundus images: an algorithm using cross-correlation of triple invariant image descriptors. IEEE Eng Med Biol. 1995;14:52-58. doi: 10.1109/51.340749. [CrossRef] [Google Scholar]

9. Cao H: A novel automated approach of multimodality retinal image registration and fusion. A PhD Dissertation, 2008

10. Chanwimaluang T: Advanced retinal imaging: feature extraction, 2-D registration, and 3-D reconstruction. A PhD Dissertation, 2006 
11. Zitov B, Flusser J, Sroubek F: Image registration: a survey and recent advances. IEEE ICIP, 2005

12. $\mathrm{Li} \mathrm{H}$, Chutatape $\mathrm{O}$. Automated feature extraction in colorretinal images by a model based approach. IEEE T Biomed Eng. 2004;51:246-254. doi: 10.1109/TBME.2003.820400. [PubMed] [CrossRef] [Google Scholar]

13. Sinthanayothin C, Boyce JF, Cook HL, Williamson TH. Automated localisation of the optic disc, fovea, and retinal blood vessels from digital colour fundus images. Br J Ophthalmol. 1999;83:902910. doi: 10.1136/bjo.83.8.902. [PMC free article] [PubMed] [CrossRef] [Google Scholar]

14. Foracchia M, Grisan E, Rugger A. Detection of optic disc in retinal images by means of a geometrical model of vessel structure. IEEE T Biomed Eng. 2004;23:1189-1195. [PubMed] [Google Scholar]

15. Barrett SF, Naess E, Molvik T. Employing the Hough transform to locate the optic disk. Biomed Sci Instrum. 2001;37:81-86. [PubMed] [Google Scholar]

16. Ibanez MV, Simo A. Bayesian detection of the fovea in eye fundus angiographies. Pattern Recogn Lett. 1999;20:229-240. doi: 10.1016/S0167-8655(98)00137-8. [CrossRef] [Google Scholar]

17. Hunter A, Lowell J, Steel D, Basu A, Ryder R: Non-linear filtering for vascular segmentation and detection of venous beading. Technical Report University of Durham, 2003

18. Delibasi KK, Kechriniotis AI, Tsonos C, Assimakis N. Automatic model-based tracing algorithm for vessel segmentation and diameter estimation. Comput Meth Prog Bio. 2010;100:108-122. doi: 10.1016/j.cmpb.2010.03.004. [PubMed] [CrossRef] [Google Scholar]

19. Gregson PH, Shen Z, Scott RC, Kozousek V. Automated grading of venous beading. Comput Biomed Res. 1995;28:291-304. doi: 10.1006/cbmr.1995.1020. [PubMed] [CrossRef] [Google Scholar]

20. Akita K, Kuga H. A computer method of understanding ocular fundus images. Pattern Recogn. 1982;16:431-443. doi: 10.1016/0031-3203(82)90022-X. [CrossRef] [Google Scholar]

21. Ramli, R., Idris, M. Y. I., Hasikin, K., A. Karim, N. K., Abdul Wahab, A. W., Ahmedy, I., ... Arof, H. Feature-Based Retinal Image Registration Using D-Saddle Feature. Journal of Healthcare Engineering, 2017, 1-15. doi:10.1155/2017/1489524

22. Hernandez-Matas, C., Zabulis, X., Triantafyllou, A., Anyfanti, P., \& Argyros, A. A. Retinal image registration under the assumption of a spherical eye. Computerized Medical Imaging and Graphics, 2017. 55, 95-105.

23. Gharabaghi, S., Daneshvar, S., \& Sedaaghi, M. H. Retinal Image Registration Using Geometrical Features. Journal of Digital Imaging, 2012, 26(2), 248-258.

24. Li, Z., Huang, F., Zhang, J., Dashtbozorg, B., Abbasi-Sureshjani, S., Sun, Y., ... Tan, T. Multi-modal and multi-vendor retina image registration. Biomedical Optics Express, 2018. 9(2), 410-418.

25. Araki, T., Ikeda, N., Dey, N., Chakraborty, S., Saba, L., Kumar, D., Suri, J. S. A comparative approach of four different image registration techniques for quantitative assessment of coronary artery calcium lesions using intravascular ultrasound. Computer Methods and Programs in Biomedicine, 2015. 118(2), 158-172.

26. Wang, C., Ren, Q., Qin, X., \& Yu, Y. Adaptive DiffeomorphicMultiresolution Demons and Their Application to Same Modality Medical Image Registration with Large Deformation. International Journal of Biomedical Imaging, 2018, 1-9.

27. Chakraborty, S., Ghosh, S., Chatterjee, S., Chowdhuri, S., Ray, R., \&Dey, N. Rigid image registration using parallel processing. International Conference on Circuits, Communication, Control and Computing, 2014. pp.15-22.

28. Tang Z, Fan Y. Groupwise Image Registration Guided by a Dynamic Digraph of Images. Neuroinformatics. 2016. 14:131-145.

29. Chakraborty, S., Dey, N., Nath, S., Roy, S., \&Acharjee, S. Effects of rigid, affine, b-splines and demons registration on video content: A review. International Conference on Control, Instrumentation, Communication and Computational Technologies (ICCICCT), 2014. pp. 101-107. 
30. Thirion,J.P. Image matching as a diffusion process: an analogy with Maxwell's demons.Medical Image Analysis. 1998. 2(3), 243-260.

31. Gu, X., Pan,H., Liang,Y., Castillo, R., Yang,D., Choi,D., Castillo,E.,Majumdar, A., Guerrero, T., Jiang,S. B. Implementation and evaluation of various demons deformable image registration algorithms on a GPU. Phys. Med. Biol. 2010. 55(1), 207-219.

32. Papie z, B. W., Franklin, J. M., Heinrich, M. P., Gleeson,F. V., Brady, M. and Schnabel, J. A. GIFTed Demons: Deformable Image Registration with Local Structure-Preserving Regularization Using Supervoxels for Liver Applications. Journal of Medical Imaging. 2018. 5(2), pp.1-5.

33. Ayatollahi, F., Shokouhi, S.B., Ayatollahi, A. A new hybrid particle swarm optimization for multimodal brain image registration. J. Biomedical Science and Engineering, 2012, 5, 153-161.

34. Ashour, S.A., Samanta, S., Dey, N., Kausar, N., Abdessalemkaraa,B.W., Hassanien,E.A. Computed tomography image enhancement using cuckoo search: a log transform based approach. JSIP, 2015. 6(3), 244-257.

35. Shehab, M. Artificial Intelligence in Diffusion MRI. Studies in Computational Intelligence. 2020. vol. 877, pp. 1-9.

36. Daniel, E., Anitha, J., Kamaleshwaran, K., \& Rani, I. Optimum spectrum mask based medical image fusion using Gray Wolf Optimization. Biomedical Signal Processing and Control, 2017. 34, 36-43.

37. Daniel, E. Optimum Wavelet-Based Homomorphic Medical Image Fusion Using Hybrid GeneticGrey Wolf Optimization Algorithm. IEEE Sensors Journal, 2018. 18(16), 6804-6811. doi:10.1109/jsen.2018.2822712

38. Zhang $\mathrm{Y}, \mathrm{Wu} \mathrm{L}$ A novel method for rigid image registration based on firefly algorithm. Int J Res Rev Soft IntellComput, 2012 2:141-146.

39. Cocianu, C.-L., \& Stan, A. New Evolutionary-Based Techniques for Image Registration. Applied Sciences, 2019, 9(1), 176.

40. Gao, Z.-M., \& Zhao, J. An Improved Grey Wolf Optimization Algorithm with Variable Weights. Computational Intelligence and Neuroscience, 2019, 1-13.

41. Mirjalili, S., Mirjalili, S. M., \& Lewis, A. Grey Wolf Optimizer. Advances in Engineering Software, 2014. 69, 46-61.

42. Yang, X.S. and He, X. Firefly Algorithm: Recent Advances and Applications. Int. J. Swarm Intelligence. 2013. 1(1), 36-50.

43. Lan, S., Guo, Z., \& You, J. Non-rigid medical image registration using image field in Demons algorithm. Pattern Recognition Letters. 2019. Vol.125, 98-104.

44. Chakraborty, S., Dey, N., Samanta, S., Ashour, A. S., \&Balas, V. E. Firefly algorithm for optimized nonrigid demons registration. Bio-Inspired Computation and Applications in Image Processing, 2016. 221-237.

45. Chakraborty, S., Dey, N., Samanta, S., Ashour, A. S., Barna, C., \&Balas, M. M. Optimization of Nonrigid Demons Registration Using Cuckoo Search Algorithm. Cognitive Computation, 2017. 9(6), 817826.

46. Mishra, B., Pati, U. C., \&Sinha, U. Modified demons registration for highly deformed medical images. 2015 Third International Conference on Image Information Processing (ICIIP). 2015, pp. 1-6.

47. Zhuang, S., \& Xia, Y. A novel deformable image registration algorithm based on improved active demons. 2017 10th International Congress on Image and Signal Processing, BioMedical Engineering and Informatics (CISP-BMEI). 2017, pp. 117-123.

48. Yogapriya, J., \& Nithya, B. Fuzzy Based Grey Wolf Optimization for Effective Medical Image Retrieval System. International Conference on Communication and Signal Processing (ICCSP). 2018, pp. $1-6$. 
49. Asha, C.S. ,Lal, S., Gurupur, V. P., \&Saxena, P. U. P. Multi-modal Medical Image Fusion with Adaptive Weighted Combination of NSST Bands using Chaotic Grey Wolf Optimization. IEEE Access, 2019. vol. 7, pp. 40782-40796.

50. Zhang, Y.D., Wu, L. Rigid Image Registration based on Normalized Cross Correlation and Chaotic Firefly Algorithm. International Journal of Digital Content Technology and its Applications. 2012. 6(22):129-140

51. Xiaogang, D., Jianwu, D., Yangping, W., Xinguo, L., \&Sha, L. An Algorithm Multi-Resolution Medical Image Registration Based on Firefly Algorithm and Powell. 2013 Third International Conference on Intelligent System Design and Engineering Applications. 2013. pp. 68-74.

52. Li, T., Li, P., Li, W., Gao, L., Yi, J., \&Bai, Y. (2015). Cuckoo Search-based range image registration for free-form surface inspection. 2015 IEEE 19th International Conference on Computer Supported Cooperative Work in Design (CSCWD). 2015. pp. 1-6.

53. Jebril, N. A., \& Abu Al-Haija, Q. Cuckoo Optimization Algorithm (COA) for Image Processing. Nature Inspired Optimization Techniques for Image Processing Applications, 2018. pp. 189-213.

54. Vercauteren, T., Pennec, X., Perchant, A., \&Ayache, N. Non-parametric Diffeomorphic Image Registration with the Demons Algorithm. Lecture Notes in Computer Science, 2007. pp. 319-326.

55. Yang, X.S., Deb, S., (2009). Cuckoo search via Lévy flights. Proc. of World Congress on Nature $\mathcal{E}$ Biologically Inspired Computing (NaBIC 2009), 2009. 210-214.

56. Ahmed S. S., Dey N., Ashour A. S., Sifaki-Pistolla D., Bălas-Timar D., Balas V. E., Tavares J. M. R. Effect of fuzzy partitioning in Crohn's disease classification: a neuro-fuzzy-based approach. Medical $\mathcal{E}$ biological engineering $\mathcal{E}$ computing, 2016. pp. 1-15.

57. del Moral R., Navarro J., Lahoz-Beltra R., Marijuán P. C. Cognitive and Emotional Contents of Laughter: Framing a New Neurocomputational Approach. International Journal of Synthetic Emotions (IJSE) 2014. 5(2): 31-54.

58. Naik A., Satapathy S. C., Ashour A. S., Dey N. Social group optimization for global optimization of multimodal functions and data clustering problems. Neural Computing and Applications, 2016, pp.1-17.

59. Roy P., Goswami S., Chakraborty S., Azar A. T., Dey, N. Image segmentation using rough set theory: a review. International Journal of Rough Sets and Data Analysis (IJRSDA), 2014. 1(2): 62-74.

60. Dey N., Ashour, A. S. Chakraborty S., Samanta S., Sifaki-Pistolla D., Ashour A. S., et al. (2016) Healthy and Unhealthy Rat Hippocampus Cells Classification: A Neural Based Automated System for Alzheimer Disease Classification. Journal of Advanced Microscopy Research, 2016. 11(1): 1-10.

61. Wang, H., Dong, L., O'Daniel, J., Mohan, R., Garden, A. S., Ang, K. K., Cheung, R. Validation of an accelerated "demons" algorithm for deformable image registration in radiation therapy. Physics in Medicine and Biology, 2005. 50(12), 2887-2905.

62. Parekar, J., Porwal, P., \& Kokare, M. Automatic retinal image registration using fully connected vascular tree. 2016 International Conference on Signal and Information Processing (IConSIP). 2016. pp. 1-6.

63. Hang, Y., Zhang, X., Shao, Y., Wu, H., \& Sun, W. Retinal image registration based on the feature of bifurcation point. 2017 10th International Congress on Image and Signal Processing, BioMedical Engineering and Informatics (CISP-BMEI). 2017. pp. 1-5.

64. Tang, H., Pan, A., Yang, Y., Yang, K., Luo, Y., Zhang S., Ong, S-H. Retinal Image Registration Based on Robust Non-Rigid Point Matching Method. Journal of Medical Imaging and Health Informatics, 2018. Vol. 8, 1-10.

\section{Web Reference}

65. https://projects.ics.forth.gr/cvrl/fire/ 